\title{
Modeling Design Objects in CAD System for Service/Product Engineering
}

Tomohiko Sakao, Yoshiki Shimomura, Erik Sundin and Mica Comstock

\section{Linköping University Post Print}

N.B.: When citing this work, cite the original article.

Original Publication:

Tomohiko Sakao, Yoshiki Shimomura, Erik Sundin and Mica Comstock, Modeling Design Objects in CAD System for Service/Product Engineering, 2009, Computer-Aided Design, (41), 3, 197-213.

http://dx.doi.org/10.1016/j.cad.2008.06.006

Copyright: Elsevier

http://www.elsevier.com/

Postprint available at: Linköping University Electronic Press

http://urn.kb.se/resolve?urn=urn:nbn:se:liu:diva-42689 


\title{
Modeling Design Objects in CAD System for Service/Product Engineering
}

\author{
Tomohiko Sakao (Corresponding author) \\ Institute for Product Development and Machine Elements, Darmstadt University of Technology \\ Magdalenenstrasse 4, 64289 Darmstadt, Germany \\ (Current affiliation: Department of Management and Engineering, Linköping University \\ SE-581 83 Linköping, Sweden \\ Phone:+46-13-282287 \\ e-mail:tomohiko.sakao@liu.se ) \\ Yoshiki Shimomura \\ Department of System Design, Tokyo Metropolitan University \\ Minamiosawa 1-1, Hachioji-shi, Tokyo 192-0397, Japan \\ Phone / Fax: +81-426-77-2729 \\ e-mail: yoshiki-shimomura@center.tmu.ac.jp \\ Erik Sundin and Mica Comstock \\ Department of Management and Engineering, Linköping University, \\ SE-581 83, Linköping, Sweden \\ Phone: +46-13-286601, Fax: +46-13-282798 \\ e-mail: erik.sundin@liu.se
}

\section{Abstract:}

This paper proposes a new type of service CAD system utilized in Service/Product Engineering (SPE), a much-needed and novel engineering discipline within the background of servicification. In this research a design-object model was defined, and a prototype named Service Explorer was implemented. The model represents critical concepts such as value, costs, functions either of products or of service activities, and entities. Through its application to business cases such as selling washing machines, providing pay-per-wash service, and cleaning washing machines, the Service Explorer was proven to support designers as they describe and operate design objects. In the future we expect that the Service Explorer can help designers with generating new ideas.

Keywords: Conceptual Design, Product/Service System, Operation 


\section{Introduction}

It goes without saying that in the tertiary industry (i.e. the third industry), service activities are the source of core value and are regarded as crucial. It is noteworthy that manufacturers today regard service activities as increasingly important. Some manufacturing firms are shifting from a "product seller" role toward that of a "service provider" $[1,2]$. One reason from the demand side is the "servicification" of consumers' behaviors, meaning a shift from customers' consumption of physical products to consumption of softer or solution-based services. This trend can, for example, be observed in statistics of the expenditure in a household with more than one person in Japan. If this is classified into the expenditure for "goods" and "services," the latter can been seen as steadily growing, accounting for $42 \%$ in the year 2002, up from just $36 \%$ in the year 1987 [3].

In the supply side parallel to the trend above, a concept named Total Care Products (Functional Products) [4], which comprises combinations of hardware and support services, has recently been developed in the engineering domain. In addition, similar concepts such as Product/Service Systems (PSS) [5, 6] and Functional Sales [7] are seen not only in theoretical contexts, but also in practical industrial applications. Service activity is increasingly incorporated into the design space commonly across these concepts. This area has been traditionally dominated by physical products in manufacturing industries.

As a result of the trend in the demand and supply sides, it has been highlighted that product characteristics and service activities influence one another; furthermore, a component in a physical product can be an alternative for a part of the associated service activities, and vice versa. Actually, widening the design space by incorporating service design into product design is among the promising avenues for increased competitiveness. This is especially true if innovative solutions [8] are considered.

To design such products associated with services using computer systems effectively and efficiently, a different model from the traditional one is required. However, very few researchers have dealt with services from the viewpoint of design, while services have often been a traditional topic in the fields of marketing and management (e.g. [9]).

To tackle this problem, a novel engineering discipline called Service/Product Engineering (SPE) [10], formerly called Service Engineering [11], has been proposed. This paper proposes a new type of design-object model and a computerized system for representing design objects to support conceptual design in SPE.

The remainder of the paper consists of the following sections. Section 2 begins with an explanation of how different the concerned design is compared to traditional product design, and then describes the prerequisites of the CAD system. Section 3 then proposes the model of design-object and the service CAD system, while Section 4 verifies the proposed model and system through application to industrial business cases. Finally, following some discussion in Section 5, Section 6 concludes the paper. 


\section{Nature of Design and Required CAD System in Service/Product Engineering}

\subsection{Nature of Design in SPE}

In this research, a "service" is defined as "an activity that a provider causes a receiver, usually with consideration, to change from an existing state to a new state that the receiver desires, where both contents and a channel are means to realize the service" [11] (see Fig. 1). Service contents are provided by a service provider and delivered through a service channel. Physical products are either the service contents or the service channel. Service activities, on the other hand, support service contents to be transferred or activate service channels. Thus, selling physical products is also regarded here as a service. Hence, a service receiver is satisfied with just contents, which are any type of material, energy, or information, while a service channel is used to transfer, amplify, and/or control the service contents.

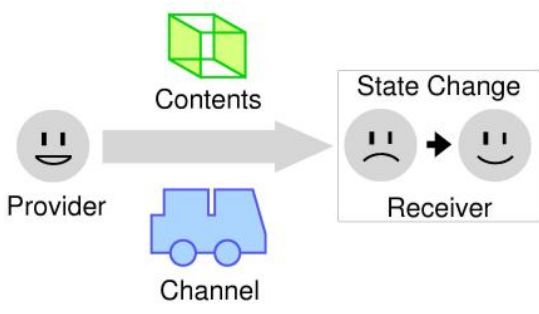

Fig. 1. The elements of service.

The term "service", already defined, is related to the concept of Service/Product Engineering (SPE), which is a discipline to increase the value of artifacts by focusing on service [10]. Note that SPE has both analytical and synthetic aspects. SPE aims at intensifying, improving, and automating the entire framework of service creation, delivery, and consumption. To increase the total satisfaction of receivers, we can improve functions and/or quality of both channels and contents. Traditionally, engineering design has aimed to improve only functions. A better function of a new product, we have believed, makes consumers satisfied. In SPE, however, not only the functions of artifacts but also the meaning of contents must be matched to the specifications given by receivers; only then will the satisfaction level of receivers increase.

As described above, the critical concept in SPE is not the function of a product, but rather the state change of the receiver. The state change can be fulfilled either by products or by service activities. In other words, the target in SPE is shifted from functions or quality to value. For instance, consider a service by a refrigerating facility provider for a logistics firm dealing with fresh meat. Maintaining the temperature in the facility is the target in this case. Developing both a refrigerating facility with low malfunction frequency and a system for technicians to visit the site quickly in case of malfunction are possible measures to fulfill the goal.

It is quite obvious that the design space given to conceptual design in SPE is wider than that available in the latter steps. The potential result of this is more design options as well as different solutions. At the same time, optimization at the conceptual design stage in SPE can prevent designers from placing too much effort on the detailed design of unimportant parts (i.e. parts that are not so crucial to the final service). 
On the other hand, such a design has much in common with conventional design. As Ramaswamy [12] suggested, service design begins with defining design attributes, followed by specifying design performance standards and generating/evaluating concepts; detailed design comes later. In addition, analysis of existing designs of services has revealed that most of them can be classified into the three types [13]: 1) (re-)design of a new service by enhancing components of and improving existing services; 2) application of an existing service to a different field; and 3) creative new design.

\subsection{Existing Research}

Regarding research on service design and development in the tertiary industry, Shostack [9] and Ramaswamy [12] have proposed methods to design and manage services effectively, but mainly for marketers in the service industry. It should be noted that the main designed object here is the activities of the employees facing the customers. Among them, Ramaswamy discusses the method to design the process after the service constituents are classified into product, facility, and process. Bullinger et al. [14] discuss the ways to manage organizations, human resources, and IT (information technology) systems for service design.

Let us look at the field of emerging business activities in the manufacturing sector, which is called PSS (Product/Service system) [5, 6] PSS is a set of products and services provided to customers. Weber et al. [15] have proposed a scheme to design and develop PSS by adding the element of a customer, who has functionality to feedback to the PSS requirements from product characteristics, in their model of traditional product design. As reviewed above, there exists neither a method nor CAD systems to design services and associated physical products applicable to the service or PSS based on the inputs of customers' requirements for the services.

Relatively more research, however, is available from the field of CAD systems for products. CAD systems often focus on specific areas in product development, and CAD systems specifically for product functions are available (e.g. [16, 17]). More dominant are CAD systems dealing with physical characteristics such as feature and geometry, including those for product manufacturing and assembly. However, only a portion of the interesting research related to this research's issues is introduced here.

CAD systems and their tools have been rapidly developed in recent years, in tandem with the development of information technology tools and the increasing use of the Internet. The trends of company globalization require that information about the product/service concepts be transferred to all parts of the world. For example, the product development department in Sweden needs to share its ideas concerning upcoming service/products with the manufacturing department in Malaysia in a convenient manner. As a result, there is clearly a great need for CAD systems for collaboration [18]. Examples of these collaborative CAD systems to meet this important need include Alibre Design, CoCreate, OneSpace, etc. [19]. At the MIT CADlab, an approach for service marketplace called DOME was developed during 1999 [20]. This marketplace consisted of three elements: providing design participants with the ability to publish live services as part of their natural workflow; creating a service market that allows system integrators to seamlessly interrelate product development services across many different 
software applications and model servers; and the introduction of tools that facilitate the rapid elucidation and evaluation of trade-offs. Interestingly, they hypothesized that product development services will become commodities, much like many component-level products are today.

Having a standard of product data able to exchange between heterogeneous modelling systems, such as CAD systems, is critical for collaborative design and manufacturing [21]. In the US, the National Institute of Standards and Technology (NIST) have set a goal "to establish a standard that supports the seamless interoperability among current and next generation computer-aided design systems and between CAD systems and other systems that generate and use products" [21]. This means, for example, that there is a need for CAD systems to be able to exchange data with Product Lifecycle Management (PLM) systems and Product Data Management (PDM) systems.

Other researchers have looked at ways for CAD systems to go from design CAD views to manufacturing views. For instance, Hoffman and Joan-Arinyo [22] proposed the use of "a product master model" in a "waterfall approach" (see Fig. 2 based on [22]). They indicated that many design changes that arise from the downstream views can be formalized by a well-defined problem on dimensional and geometric constraints.

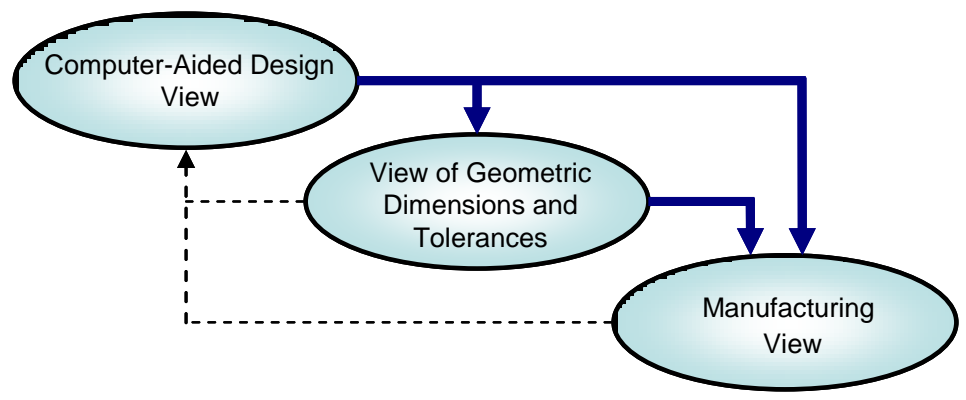

Fig. 2. The waterfall approach with information flow and feedback (based on [22]).

\subsection{Required Service CAD System}

The suggested design flow to be followed in SPE is shown in Fig. 3 (based on [10]). The first two steps, for analyzing customers and then identifying functions, correspond to conceptual design for SPE. Existing CAD systems can only cover a part of the second step. Namely, CAD systems for product functions can be applied to designing functions of products. The rest of this step, which is for designing functions of service activities as alternatives for value, cannot be supported: It is true that designing functions of service activities can be supported by some systems (e.g. [14]), however, virtually no CAD system supports design with targeting value by means of functions of products or service activities. In addition, the first step for identifying value through customer analysis is not covered either; this is exactly where a new type of CAD system is required. The two steps in the last half of this design flow are relatively well covered by the enormous number of systems and tools developed thus far. 


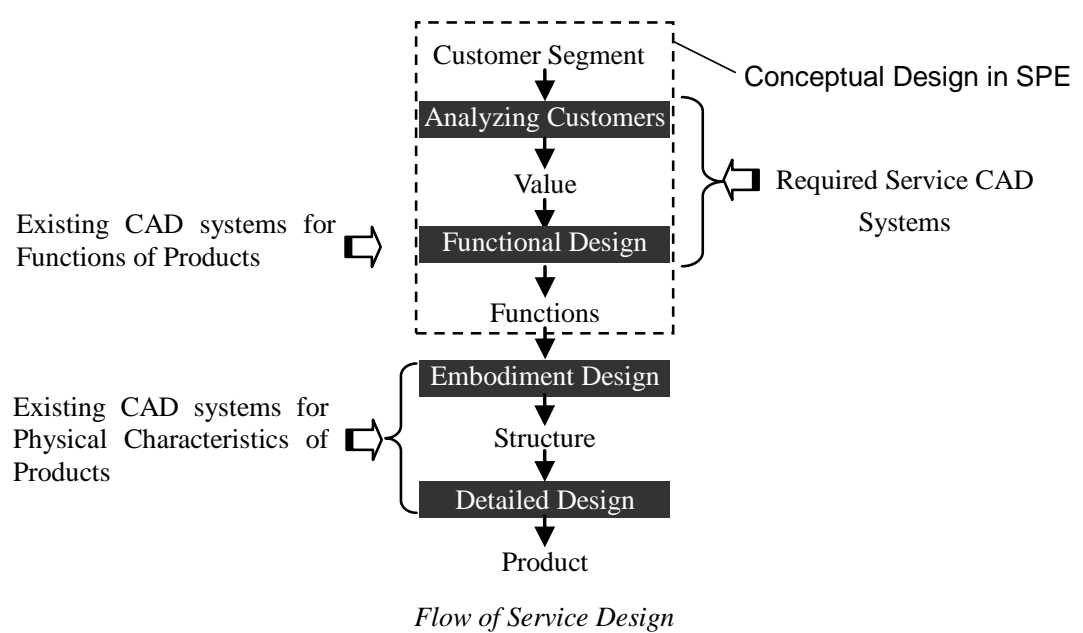

Fig. 3. Various CAD systems applied to design stages in SPE (based on [10]).

Fig. 4 depicts the constituents of the required service CAD system. In the center lies a module for "designing value" (intending provision of value): Value plays a role of interface between a provider and a receiver (recall Fig. 1). At the same time, value will be one-level more abstract than functions of products or service activities. The right-hand side shows what a service provider supplies, while what a customer demands is described on the left.

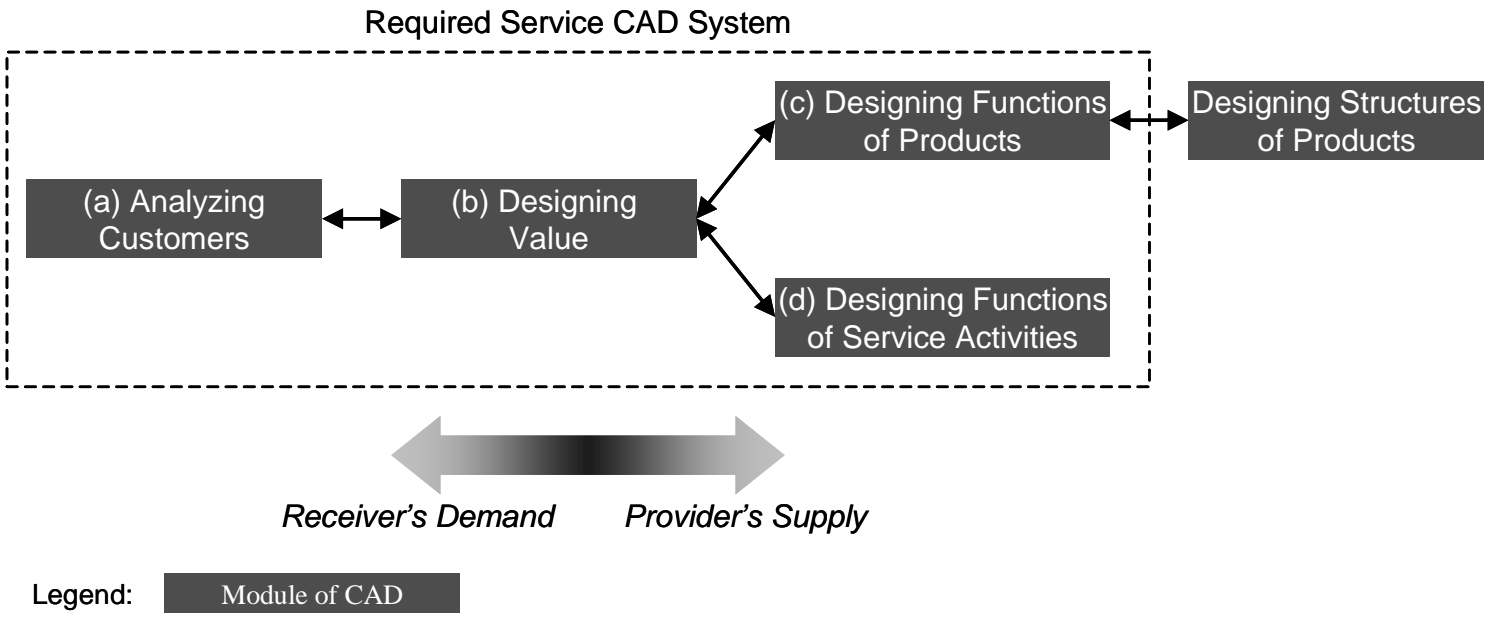

Fig. 4. Constituents of the required service CAD system.

As reviewed in Section 2.2, CAD systems supporting collaboration are increasingly demanded corresponding to globalization and recent advances in ICT (information and communication technology). Together with this trend and considering the shift from products to services in the economy, designing value is expected to be more and more important as a kernel of design in SPE. Furthermore, those kinds of existing CAD systems in Section 2.2 would preferably be integrated into this scheme. 


\section{Design-Object Model and Service CAD System}

\subsection{Model of Service}

\subsubsection{Outline}

To meet the nature of the design and the requirements for the CAD system described in Section 2, a service model has been developed consisting of four sub-models: "flow model", "scope model", "scenario model", and "view model". The remainder of Section 3.1 explains the four sub-models, after describing an important concept in those sub-models called "receiver state parameter".

The reason for modelling "receiver state parameter" (RSP) is as follows. Conventional design primarily concerns the performance of physical products; it does not consider the state change of the receiver. Designing a service must be based on the degree of satisfaction with the state change of a receiver; therefore, it is necessary to express the receiver's state changes.

Table 1 shows the information on service and how to model it in the service CAD system. The left column of Table 1 shows some fundamental elements needed to model service consisting of what, why, who, and how. This is in line with Ullman's argument on an ideal mechanical CAD system [23]; he argued that such a CAD system should represent not only the architecture and the shape of a product but also their surrounding information such as requirements, issues, plans, and design intents. Those four elements are modeled using the scheme of RSP, scenario model, flow model, and view model. As described in Section 2, conventional design have focused on how, especially on physical products.

Table 1. Information on service and modeling schemes in the service CAD system

\begin{tabular}{ll} 
Information & Modeling scheme \\
\hline What: provided value/cost & RSP \\
\hline Why: reasons for value & $\begin{array}{l}\text { Scenario model } \\
\end{array}$ \\
& - State transition of a service receiver \\
& - Persona model of a service receiver \\
Who: $\quad$ provider and receiver & Flow/scope model \\
\hline How: measures to provide value & $\begin{array}{l}\text { View model } \\
\end{array}$ \\
& - Functions of physical products and service activities \\
& - Physical products and service bodies as entities \\
\hline
\end{tabular}

\subsubsection{Receiver State Parameter}

Receiver state parameters (RSP) [24] are classified into value and cost, depending on whether the customers like them or not. Associated sacrifice (any non-preferred change such as money, time, space, and labor) when the service is received is cost; e.g. time that customers pay for in order to walk to a launderette. The term "value" here is different from that in Value Engineering [25], where it is defined as function over economic cost. In SPE, value is defined as the change of a receiver's state that he/she prefers, so that function is just a realization method to provide the value in SPE.

Change of a receiver is represented by a set of receiver state parameters (RSP). Since an RSP 
consists of quantitative value, including Boolean logic and multi-value logic, we can compute any comparison between two RSP. In addition, we introduced a new assumption that all RSP are observable and controllable. This assumption has been unproven with human receivers because we have not had a reliable method to measure the consumer behavior. Although this assumption is quite strong, it is necessary for description of a view model (see Section 3.1.6). If it were not for this assumption, the link between RSP and a function in a view model would be meaningless for designers.

RSP change by received contents. Hence, we assume that contents consist of various functions, whose name is Function Name (FN), whose operating objects are Function Parameters (FP) and whose effect is represented by Function Influences (FI).

As the receiver's states may change with respect to supply of contents, RSP can be written as functions of contents. Parameters expressing contents are called content parameters $(\mathrm{CoP})$. In the same way, the parameters of channel, which make the flow of CoP change and thus influence RSP indirectly, are called channel parameters (ChP). Hence, we assume that both contents and channels consist of various functions, and both $\mathrm{CoP}$ and $\mathrm{ChP}$ belong to FP.

Metrics for quality/performance of service which appear in some SLAs (service level agreements), such as the availability of the server's operation in a communication providing service, should be included in the set of RSP, since the designers are supposed to meet the requirements on that quality or service. On the other hand, all the RSP are not necessarily included in such quality/performance of a service, since designers can target other parameters as well.

It should be pointed out that the selection of contents and channels is subjective. Namely, although the ways contents and channels are picked up from a service are in many cases identical, what a modeler regards to be content may be viewed as channel by another something which appears to be among the greatest reasons for services not being sufficiently dealt with as an engineering issue.

For instance, in a service performed by a cafe, one can recognize preferred RSP such as the taste of the coffee, the music, and even the available workspace. On the other hand, non-preferred RSP in the service may include a monetary cost, transportation to the cafe, and noise.

\subsubsection{Flow Model}

One of the traditional and typical services is a travel bureau, which arranges and purchases various tickets on behalf of customers. Contents are different from one ticket to another, even if all the tickets are delivered to the customers. In this way, services can be delivered through complex multiple structures consisting of various go-betweens. The intermediate agents have double characteristics of a receiver and a provider, which is represented by the symbol of the smiling circle called the "intermediate agent".

When we focus on the relationship between a receiver and a provider, many intermediate agents exist among them. We call the sequential chain of agents a "flow model" of a service [24]. Fig. 5 takes an example from a cafe service including agents such as a cafe runner, a 
customer, and a coffee machine maker. This model is needed because in SPE, designers are supposed to consider how organizations participating in a concerned service can be successful in their business. In the cafe service, the cafe runner provides the customer with drinks as well as a seat with a table. The runner needs machines to make drinks such as coffee; thus, a coffee machine maker and a furniture maker provide such instruments with the runner.
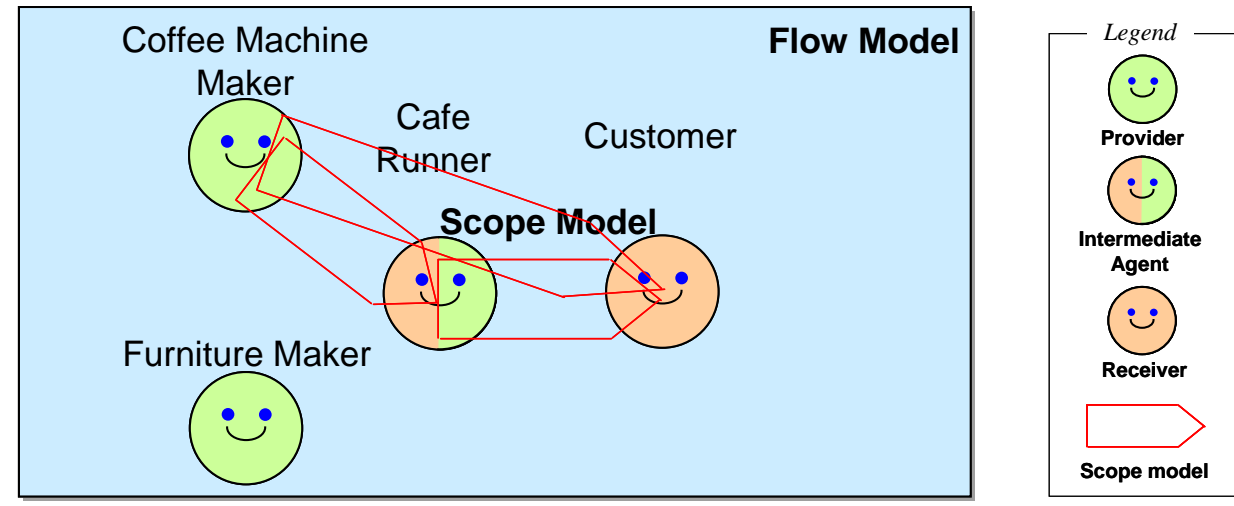

Fig. 5. The flow model and scope model.

\subsubsection{Scope Model}

Practical services have complicated structures of intermediate agents, which are connected to one another in an almost infinite chain. Therefore, it is necessary to specify the effective range of the service from an initial provider to a final receiver. For this reason, we employ a scope model between any couple of two agents that has the relation of providing/receiving value including when they do not have a direct business contract.

Fig. 5 shows instances of scope models in the cafe service. The strategy of the runner in this service can be high economic payment per a single customer responding to the desired value of the customer, with decreasing the payments to the coffee machine maker and the furniture maker. The coffee machine maker can strategically try to receive high payment from the cafe runner by meeting the requirements of the runner and of the customer. Scope models can help designers to make clear strategies, as well as to grasp the value provided/received.

\subsubsection{Scenario Model}

This model represents receivers themselves and their behaviors in receiving the service [10] for designers to analyze customers (corresponding to (a) in Fig. 4). This is necessary because the grounds behind RSP of service receivers should be understood. This is in line with the formalization of value by Bolton and Drew [26] that value is function of quality, sacrifice and customer characteristics.

A scenario model represents two kinds of information; one is a behavior of a receiver, while the other is a property of the receiver. The former is described as a transition graph whose node represents a state of a receiver and whose arc refers to a transition between two states (either temporal or causal). The upper part of Fig. 6 depicts the state transition. A state is described as a set of parameters with their values. Each transition has some parameters (called important state 
parameters) whose values change from one value to another. Furthermore, those parameters have a hierarchical relation among themselves from abstract to specific ones. By using this transition graph, the receiver's final goals and activities can be described. RSPs are a partial set of parameters of state parameters selected by designers.

This model allows designers to analyze receivers and to find appropriate RSP in the following two ways. The first one is to search a transition with no related RSP. In the case shown in Fig. 6, a transition $t_{3}$ has no RSP related. This situation suggests that designers should select a state parameter related to the transition as RSP, since the design of service/product based on this situation would not guarantee the needed state transition of receivers. In Fig. 3, the design according to only the two RSP $p_{1}$ and $p_{4}$ would not make the transition $t_{3}$ happen, and a state parameter, e.g. $p_{5}$, should be selected as RSP. On the contrary, for screening RSP in case of more RSP than necessary, relative importance of RSP can be collected from receivers and analyzed. The other way is to investigate the appropriateness of choosing as RSP another parameter in the upper or lower level of a given RSP within the hierarchy. Considering that $p_{1}$ is selected as RSP in Fig. 6, $p_{0}$ and $p_{2}$ are candidates for newly-identified RSP. Because $p_{0}$ is more abstract than $p_{l}$, identifying $p_{l}$ as RSP can lead designers to widen the coverage of receivers' needs/wishes. On the other hand, picking up $p_{2}$ is toward designing service/product responding to more specific needs/wishes. Designing value, corresponding to (b) in Fig. 4, begins with the above-mentioned process.

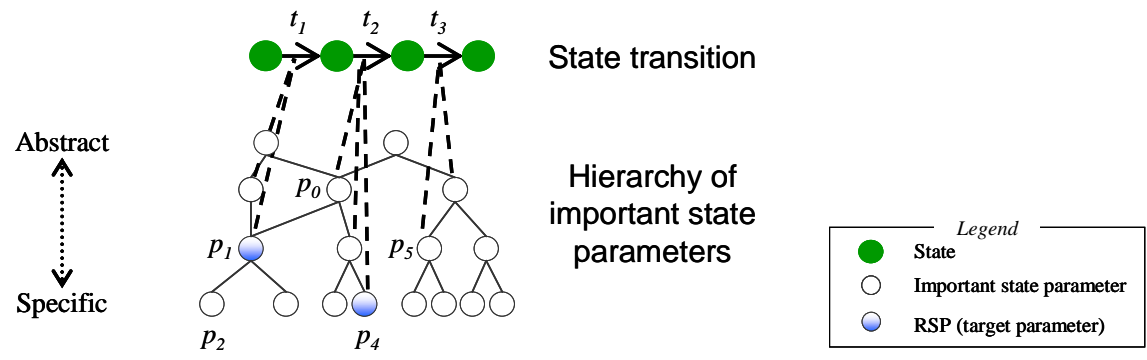

Fig. 6. Scenario model.

The latter is represented by an application of the concept called Persona [27, 28]. Persona is an imaginary target user, and is frequently used in practical design of software interfaces. The information to identify Persona is classified into two types; one is demographic data, such as age, gender, and professional carrier, while the other is psychological data, such as personality and lifestyle.

\subsubsection{View Model}

A view model [24] expresses the relationships among the elements of the service; i.e., the mutual relationships among the RSP and FP (CoP and ChP). This is where the concept of function addressed in CAD for product functions can be matched; functions of products in a view model correspond to the functions in CAD for product functions. It should be noted that not only product functions, but also those of service activities, are represented here by $\mathrm{CoP}$ and ChP. Furthermore, physical structures are represented using function parameters. Thus, some of 
the FP can be linked to geometric parameters which geometric CAD systems deal with. It should be emphasized here that a view model can work as a bridge between value represented in the form of RSP and physical structures.

Fig. 7 depicts a simple example taken from a cafe service for the customers working there. RSP change according to how the receiver evaluates subjectively the received contents. The subjectivity is crucial, and this is why the sub-model is called view model; this represents how the service receiver views the service. An RSP is linked to several CoP because the receiver evaluates the contents. The $\mathrm{CoP}$ may be supported by several $\mathrm{ChP}$ existing in the chain of several agents in the flow model. The view model illustrates visually the relationships among the parameters (RSP, $\mathrm{CoP}$, and $\mathrm{ChP}$ ) in the graph of connected nodes. The function in the middle, "Decrease Dirt on Table", is a function of service activities linked with an entity named "Waitperson". Other two functions, "provide platform to write on" and "provide place to sit on" are functions of physical products. As shown in this instance, designing functions of products and service activities (corresponding to (c) and (d) in Fig. 4) is supported with a view model. Note that the legend in Fig. 7 is also applied to Fig. 14, 18, and 19.

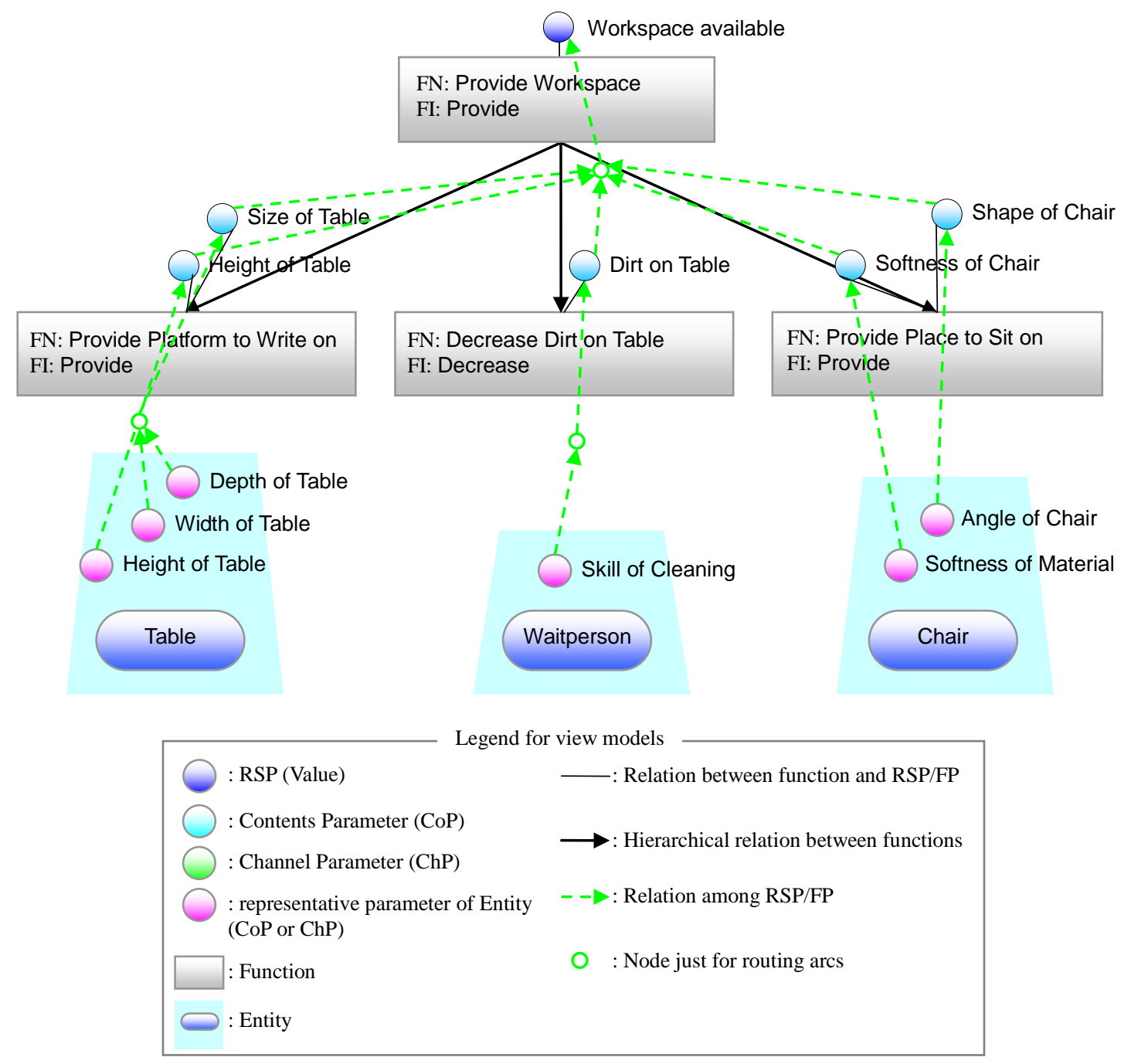

Fig. 7. A simple example of a view model. 


\subsubsection{Score on RSP}

Let us assume that it is possible to measure the degree to which a receiver recognizes each value or cost. In other words, relative importance on each value/cost can be given by a receiver. Methods to obtain such scores on RSP include direct rating and use of AHP (Analytic Hierarchy Process) [29]. Thus, service designers are able to judge the relative importance of concerned RSP. For instance, in a service by a cafe providing coffee and working place against a fee of coffee, several RSP can be rated; 5 for the taste of coffee, 3 for the working place, and 2 for the price by a receiver. Note that only the last RSP, the price of coffee, is a non-preferred RSP, namely cost.

Due to the higher level of subjectivity in a service [24], this score on RSP is well likely to vary from one receiver to another. Thus, a distribution on this score can be defined from the receivers' evaluations of a RSP.

Another stronger assumption could be introduced; how much each value/cost is for a receiver can be given. For instance, conjoint analysis [30], which is adopted widely in marketing to calculate a utility (part-worth) of a level for an attribute of products or services, can be employed for quantifying value or cost. While this assumption is quite strong, such scores are more informative. For example, a set of scores can be 2 Euro for the taste of coffee and 1 Euro for the working place, where the receiver is motivated to put twice more value on the former than the latter. We cannot derive this type of implication from the scores obtained from direct rating or AHP, as described above. Thus, the score on RSP is a strong metric to analyze customers (denoted as (a) in Fig. 4).

In either assumption, the score on RSP (denoted as $s$ ) can be formulated as follows.

$s=\mathrm{f}$ (RSP, CoP, Persona, Transition),

where RSP is the concerned RSP, CoP is a vector of the value of the concerned multiple CoP, Persona stands for the characteristics of the receiver, and Transition is for the concerned part in the state transition of the receiver. It should be noted that $s$ represents a kind of the magnitude of value or cost modeled by RSP. In addition, ChP is not included in the input, because it affects the RSP only indirectly. The formula suggests that how important an RSP is depends on the state transition to be achieved and the characteristics of the receiver and the received contents.

The scores of RSP are further used when $\mathrm{CoP}$ and $\mathrm{ChP}$ (design parameters of products or services) in a view model are determined; parameters with strong influence on the more important RSP should be handled with more effort than other parameters. Such relative importance of parameters can be obtained [31] mathematically using relative importance of RSP by taking advantage of the QFD (Quality Function Deployment) [32] method.

\subsubsection{Relations between Sub-Models}

Fig. 8 depicts the relations between the four sub-models with RSP and states of a receiver. As shown at the top of Fig. 8, a flow model represents agents participating in providing the service, while a scope model is used just for focusing on a specific combination of agents. A scope model includes all the RSP handled between the provider and the receiver [24]. Each RSP is always associated with a view model, as shown in the middle of Fig. 8. 
An RSP is grounded on the concerned receiver through a scenario model. Namely, an RSP is a part of a hierarchy of important state parameters in a scenario model, and is related to any transition between two states in a state transition of the receiver as depicted in the right-hand side of Fig. 8. As an RSP is the information on a receiver and functions and entities in a view model are that on a provider, a view model has information on both a receiver and a provider (see the bottom of Fig. 8).

Hence, the set of sub-models allow designers to describe the fundamental elements of a service and the relations between them, i.e., who provides what with whom (flow model, scope model, and RSP) with how (RSP and view model) and why (RSP and scenario model) information.

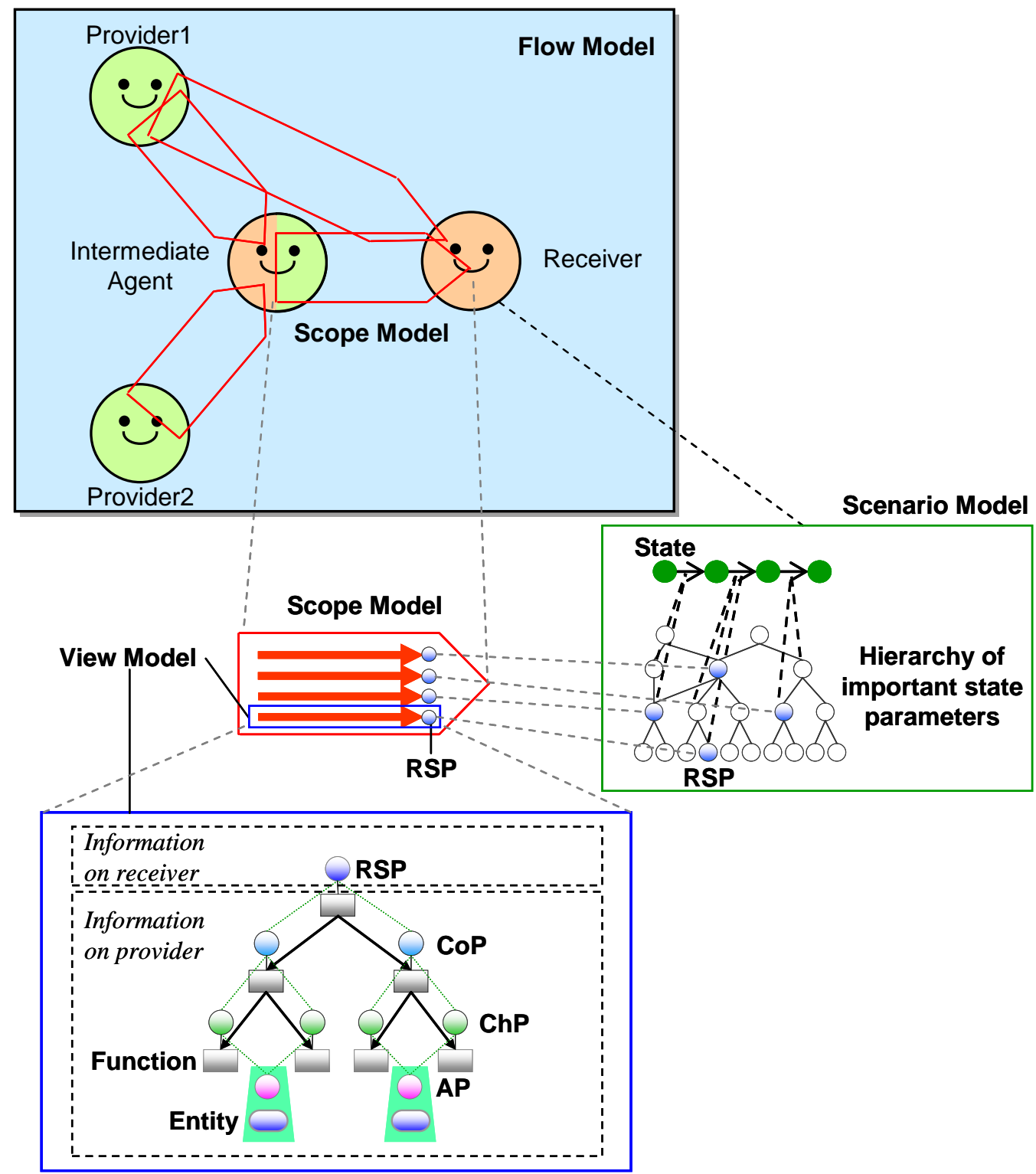

Fig. 8. The relation among the four sub models; flow, scope, scenario, view models. 


\subsection{Required Functionality for a Service CAD System}

According to the characteristics of the service design in Section 2, the CAD system is required to facilitate the following functionality.

a) Model building.

It is necessary that the service CAD system has the following five types of functionality. Required specifications and implemented ones in detail are as follows.

1) To input and edit a service model.

This is the most fundamental functionality of the service CAD system. In order to acquire the knowledge of service cases for service design in service CAD efficiently, it is necessary to provide the interface to describe a service model with ease. The knowledge of service cases means how the service is structured as a business, including who provides whom with what and how. To do so, the service CAD system should have a graphical user interface. In addition, a service model is described as a graphical structure constituted by nodes and arcs.

2) To display the component elements that designers focus on.

It is effective to be able to display (or not to display) the component elements selectively, depending on designers' demands so that they can understand the structure of the service efficiently. To do so, the service CAD system provides the function to display (or not to display) the function topology and the parameter structure.

3) To register service models in a service database.

On building a service database, it is desirable that the service database can store service models independently of the specific OS/application. For the sake of this, on the service CAD system, XML is adopted as a database description language. Herewith, input and edited service models are stored in a service database after being converted into XML data.

4) To search the service database.

To support service design by using knowledge of existing service cases, it is necessary to be able to search the service database depending on designers' requests. On the service CAD system, designers should be able to search the service database for service models with keywords contained in the composition elements (RSP, FN, FP, FI, and Entity).

5) To reuse the service model data stored in the service database.

This is the function to reuse the elements or the structure of a service model stored in the service database, when designers input and edit a service model.

b) Design guidance.

The service CAD system should be able to guide designers based on the design methodology previously mentioned.

c) Reasoning.

Several types of reasoning engines shall be stored. This functionality is crucial from the viewpoint of the information system, because designers can take good advantage of computing power. One of the promising reasoning is abduction [33, 34]. A generic computer system employing abduction named Universal Abduction Studio (UAS) [35] has already been constructed. With this type of reasoning module plugged in, the service CAD system will be able to help designers with creative design using knowledge in different business fields. 


\subsection{Prototype of Service CAD System: Service Explorer}

Based on the functional specifications mentioned in Section 3.2, the authors have developed a prototype system called Service Explorer with Java and XML. Fig. 9 depicts the system configuration of Service Explorer. The current version was developed using Java2 SDK, Standard Edition 1.4.1 and XML version 1.0.

Service Explorer includes the following modules.

1) Several databases: Databases of elements (flow/scope, scenario, and function) for existing service cases

2) Design rule base: A database of operational rules for service design

3) Service design organizer: A module to support service design processes based on a specific design methodology by means of calling other components, etc.

4) Concept rudder: A module to select a better way to display the databases to designers depending on the stage of design

5) Service evaluator: An evaluation module to evaluate a service design solution [31]

6) Reasoning engines: Reasoning engines reason about various properties of service such as similarity. A pluggable mechanism is employed so that a necessary reasoning engine is selected based on the designer's request. A reasoning engine for abduction [35] will be stored in the near future.

7) Data entry modules: Modules used when data or rules are input to those data bases or the design rule base

8) Service design working space: Temporal space storing some information retrieved or input

9) Designer interface: Interface used by designers to evaluate the service and to develop flow/scope, scenario, and view models 


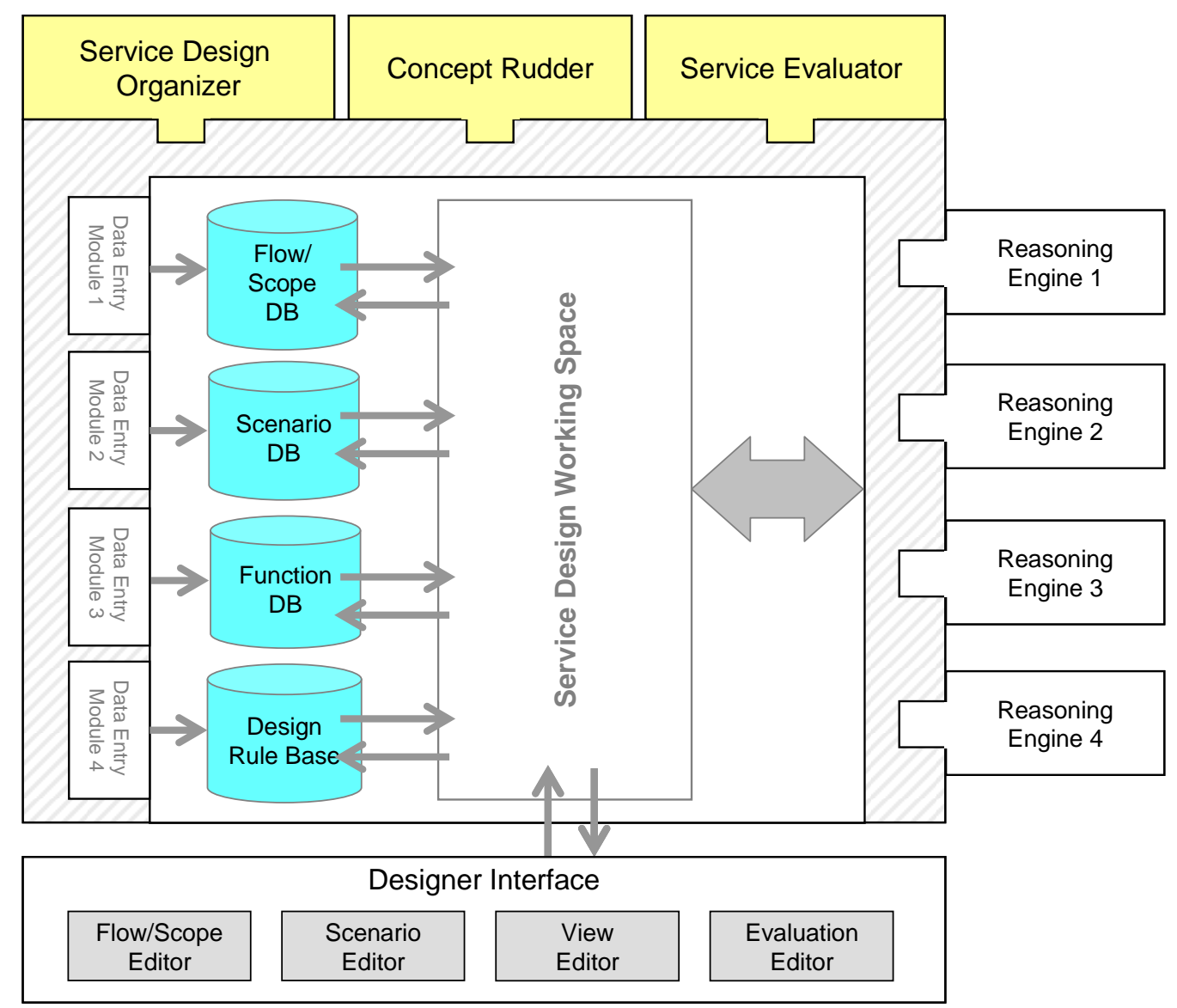

Fig. 9. Conceptual structure of Service Explorer

Fig. 10 shows the central interface of Service Explorer. In the left-hand side, users can select intended data stored in the databases through the concept rudder. Users can select one in the Persona DB to jump to edit the scenario model for the Persona. The right-hand side shows the specific model that is being built. In the upper half, users can edit the flow/scope model, while the lower half shows the information of the scope model selected in the upper half. Such information is the two agents in the scope model, the activities of the provider and the receiver, and the RSP in a state transition. Users can jump to a window for editing a view model from this interface using a pop-up menu, as shown at the bottom of Fig. 10. 


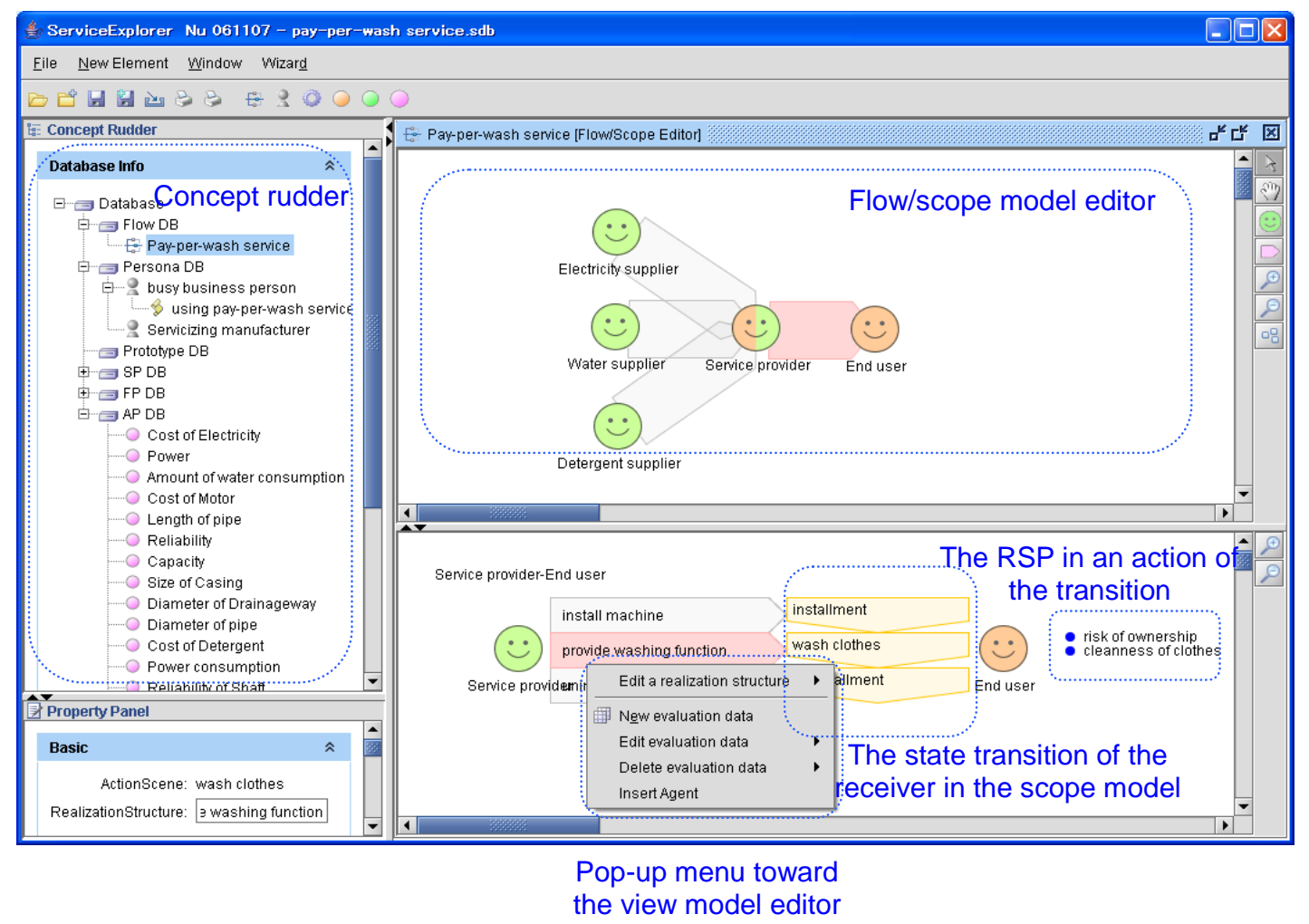

Fig. 10. Screen dump of the central interface of Service Explorer.

Designers can describe services, and register them in a database, reload them from scratch or use select pieces of data in the DB. Namely, they construct flow models, scope models, scenario models, and view models. Fig. 11 shows standardized procedures for describing models. It should be noted that designers are free to describe the scenario model first, and then the flow/scope model, instead of the order suggested in Fig. 11.

Since models (especially view models) on Service Explorer can become fairly large, we have implemented different modes of displaying view models on Service Explorer. As shown in Fig. 12, users can select the modes of displaying without functions (b) or without FP (c). By doing so, they can see, edit, and understand a view model with greater ease. 


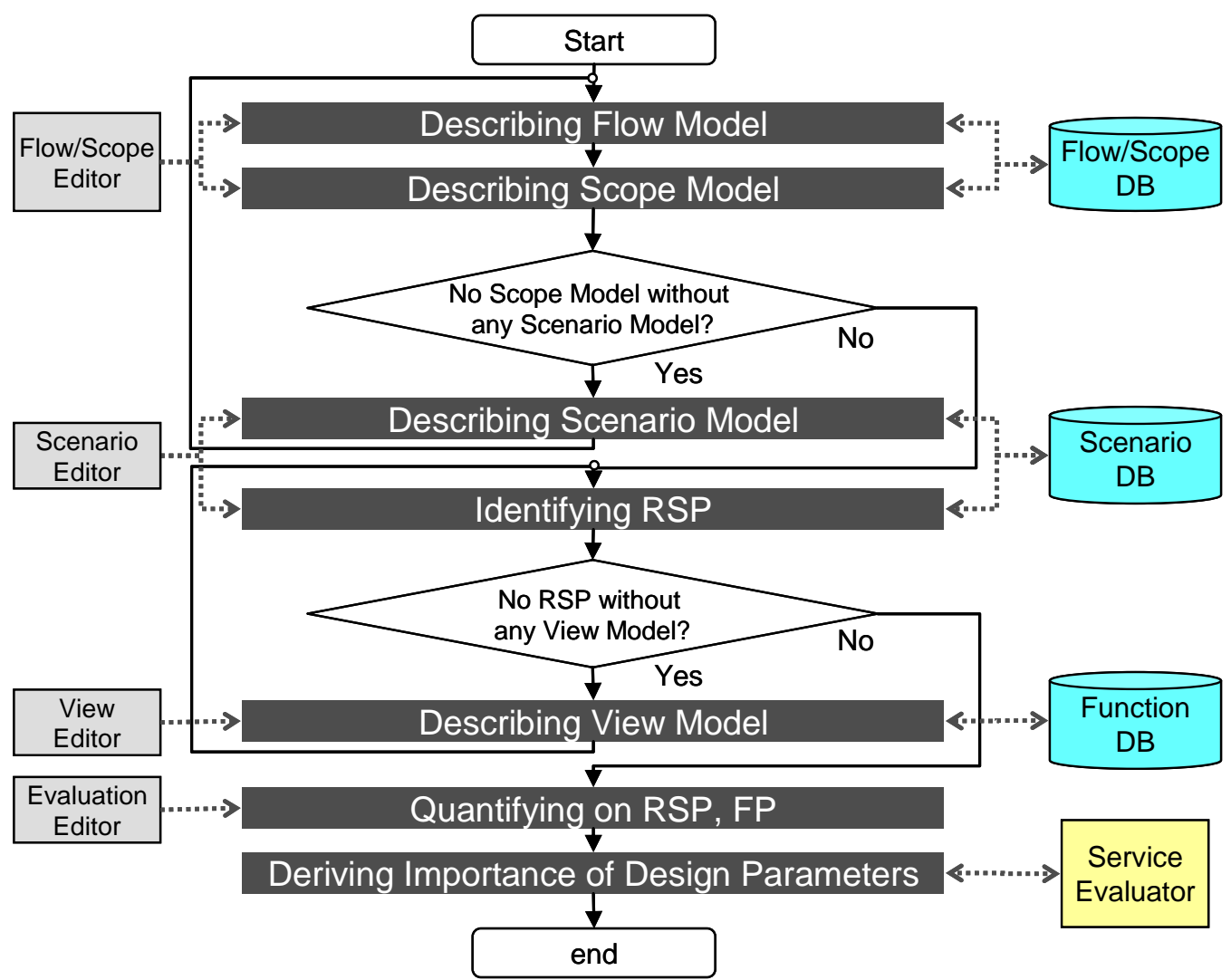

Fig. 11. Procedures for describing models on Service Explorer. 


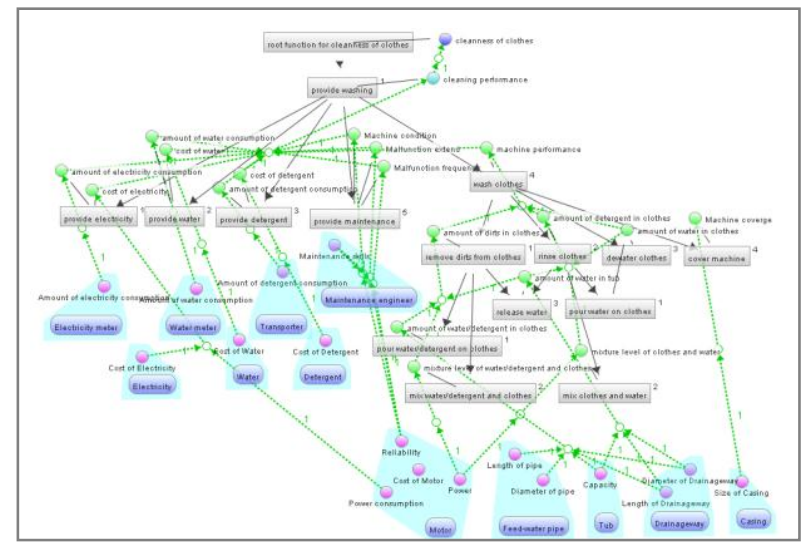

(a) display by default

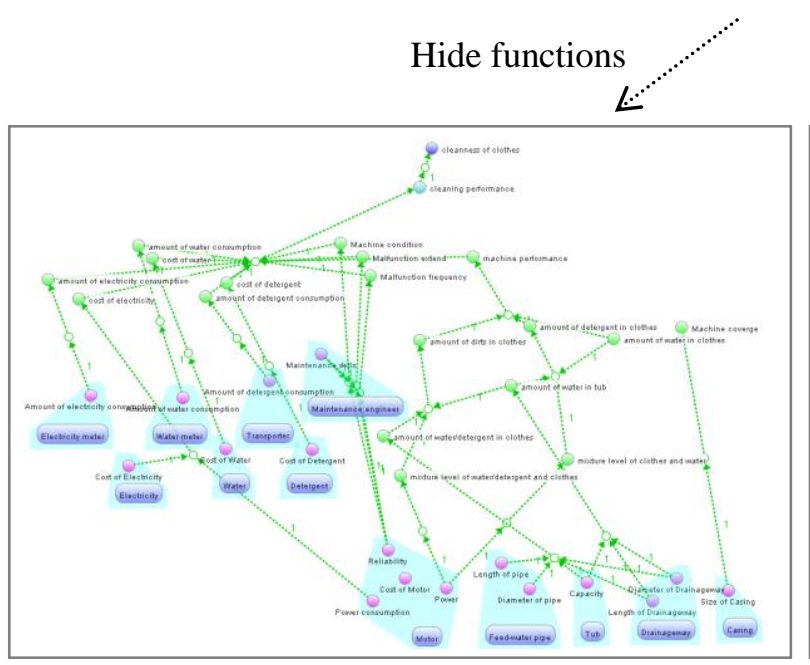

(b) display without functions
Hide FP

$\rightarrow$

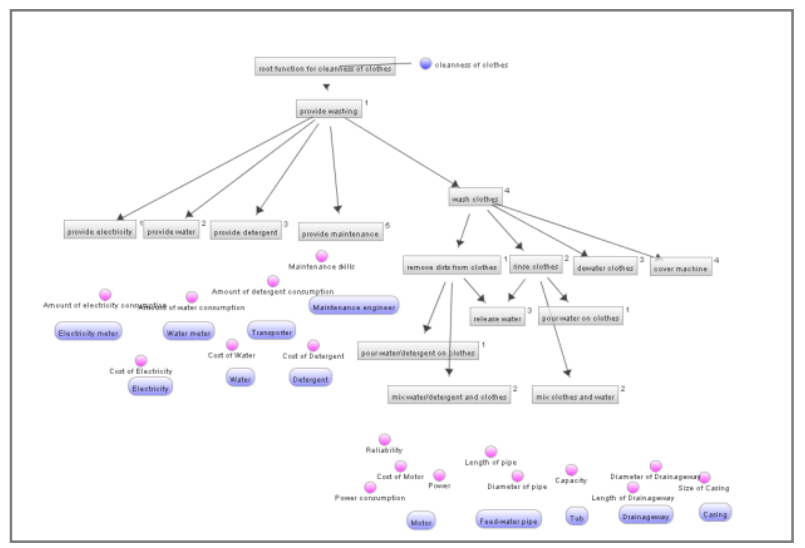

(c) display without FP

Fig. 12. Different modes of displaying view models on Service Explorer.

\section{Applications for Services Using Washing Machines}

Section 4 aims at illustrating the power of Service Explorer to describe the critical factors of services using physical products. The examples taken here all utilize a washing machine. The first instance in Section 4.1 is selling washing machines, which is the major and the traditional way of providing the function of washing machines. The next one in Section 4.2 is a hypothetical pay-per-wash service, which the authors conceptualized after several services with washing machines in Sweden and Japan. The final example in Section 4.3 is a cleaning service of washing machines, modeled after a service available in Japan.

\subsection{Selling Washing Machines}

The flow model described in Service Explorer includes the washing-machine manufacturer (described as "machine manufacturer"), the distributing retailer, ("retailer"), and the end receiver ("end user") (see Fig. 13). The Persona of the end receiver is set to be a so-called price buyer, who cares about the price of physical products but very little for other factors. 


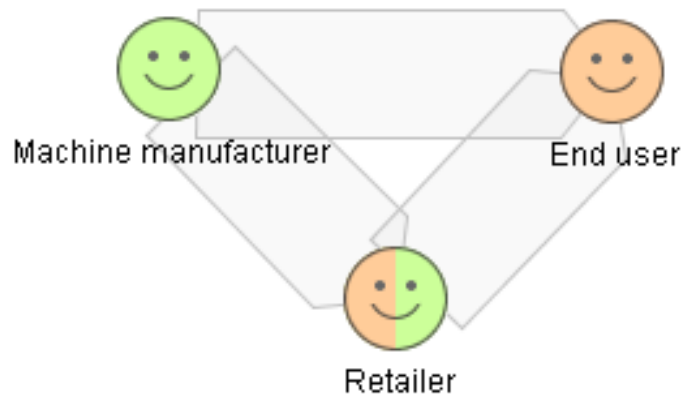

Fig. 13. Flow model of this service.

The RSP described in each scope model and its view models representing the realization structures are as follows.

- From the machine manufacturer to the end receiver

The value of the end receiver was described with an RSP named "machine performance", while the economic cost upon purchase was described with the other RSP (cost RSP). The realization structure to affect the "machine performance" was described by the view model in Fig. 14. Namely, this RSP was associated with the function of "root function for machine performance", which is automatically generated by Service Explorer. This is deployed into "remove dirt from clothes", "rinse clothes", "remove water from clothes", and "cover machine". The CoP describing these functions include "amount of dirt in clothes" and "amount of water in clothes". Some of these functions are further deployed into lower functions such as "mix water/detergent and clothes". At the bottom, ChP are linked with AP describing entities; e.g., the ChP named "mixture level of water/detergent and clothes" is linked with the AP named "power" of "motor". Other described entities are "feed-water pipe", "tub", "drainage way", and "casing". As a whole, this view model means the machine performance, the concerned RSP, is provided a set of those physical entities such as a tub. It should be noted that all the functions are the functions of physical products, since this service does not include service activities.

It should be emphasized that the AP of the motor, "Reliability" and "Power consumption" (strongly linked to electricity cost paid by the end user), are not linked to other parameters in the upper part of the view model. This means that the RSP, which is desired by the price buyer, is not related to these AP. Thus, designers can understand that the reliability and the power consumption of the motor are not important parameters in this case.

- From the manufacturer to the retailer

The value of the retailer is the washing machine, while the space for displaying washing machines was described as cost RSP, which is described to have a relation to an FP for the washing-machine size.

- From the retailer to the end receiver

The information that the end receiver receives from sales persons at the retailer upon the buying decision was modeled as value, while the transport of the purchased product was described as a cost RSP. 


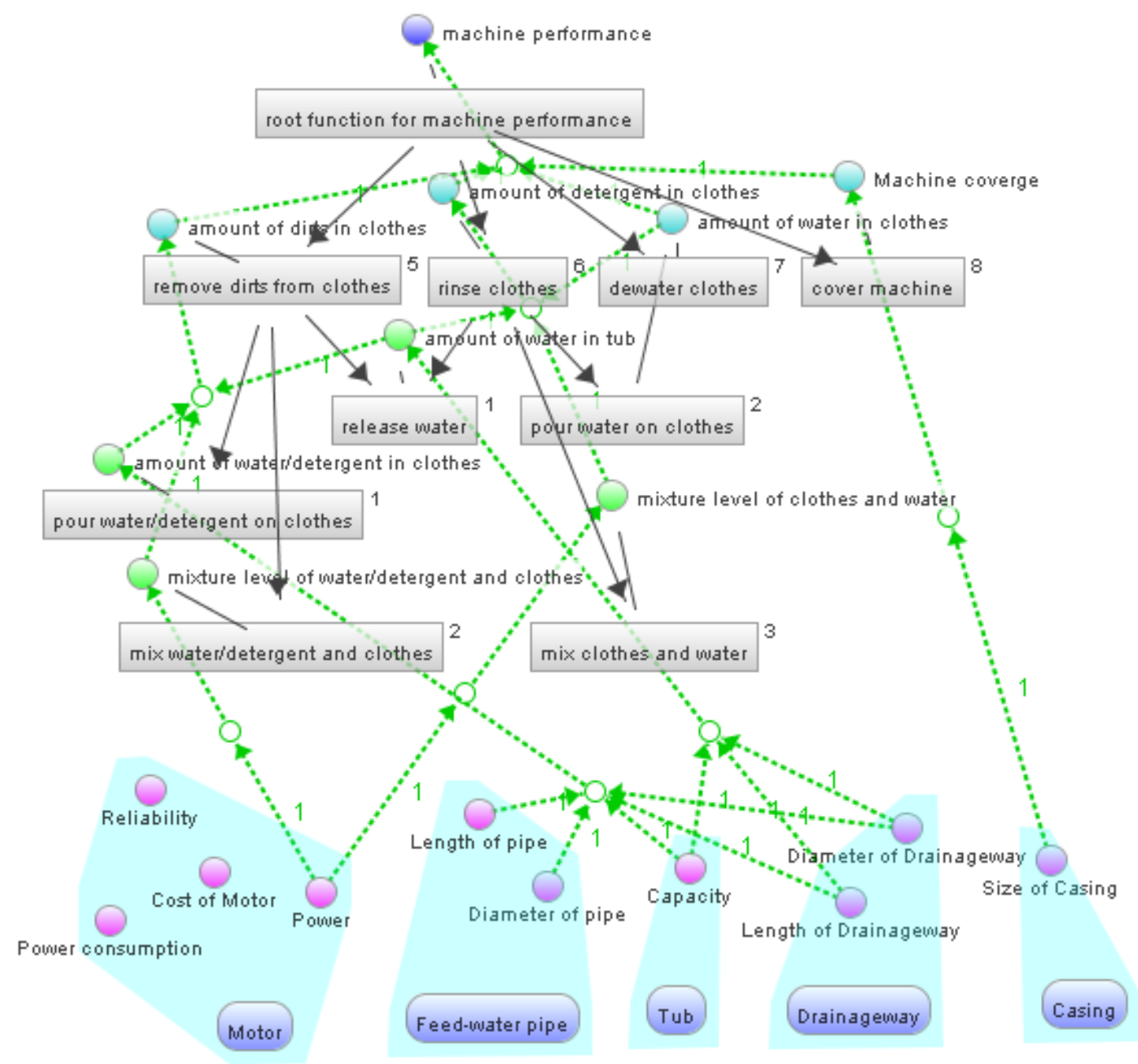

Fig. 14. The view model for cleanness. 


\subsection{Pay-per-wash Service}

In this service, an end receiver (i.e. washing-machine user) rents a washing machine at his/her place of living for a certain contracted period of time, and pays a fee per wash (e.g. 1 Euro / 1 wash) to the service provider. The provider carries out maintenance when the machine malfunctions. In addition, the fee includes the electricity, water, and detergent used in the washing process. The main benefits for the end receiver include reduction of economic costs (if the rental period is relatively short) and risk when compared to the selling service described in Section 4.1. For instance, the end receiver has no risk from machine malfunctions and is not obliged to bear the EOL (end-of-life) cost when the machine is disposed. This is a new potential market worth being investigated by washing-machine manufacturers.

It is quite critical for designers to grasp the Persona of the end receiver in this service. Designers described it in the Persona editor as shown in Fig. 15. This allows designers to share the concrete and accurate picture of the target receiver; for instance, the texts in "Personality" tell designers that time is very precious for the target receiver, and the LOV (list of values) shows his three relatively important values such as "being well respected".

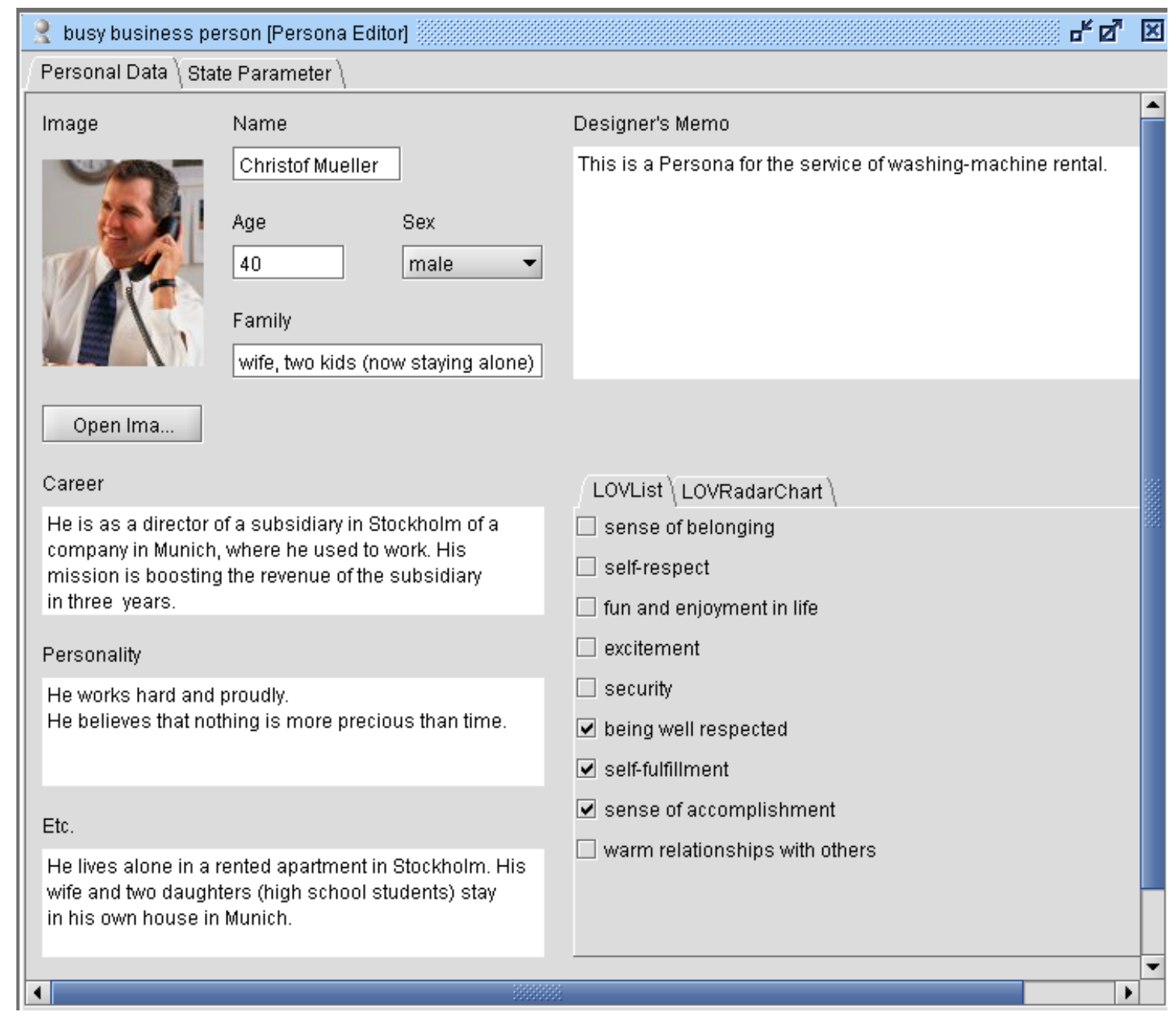

Fig. 15. A screen dump of description of Persona model on Service Explorer. 
Based on the Persona, the state transition of the end user was described as shown in the upper half of Fig. 16. It begins with the installment, followed by washing clothes in daily life, and finally the uninstallment.

Designers described a hierarchy of important parameters for the Persona for the whole state transition. In addition, they identified to which action (a piece of transition) in the state transition those parameters should be related. Then, they selected some of the state parameters as RSP (the target design parameters). As shown in the lower half of Fig. 16, in the action, "wash clothes", "cleanness of clothes" and "risk of ownership" are selected as RSP from the hierarchy of the important parameters. It should be noted that the upper one of "risk of ownership" is "time" and followed by "being well respected", while the lower one is "risk of malfunction". In addition, the other RSP, "cleanness of clothes", is deployed into "machine performance" and "cleanness of washing machine".

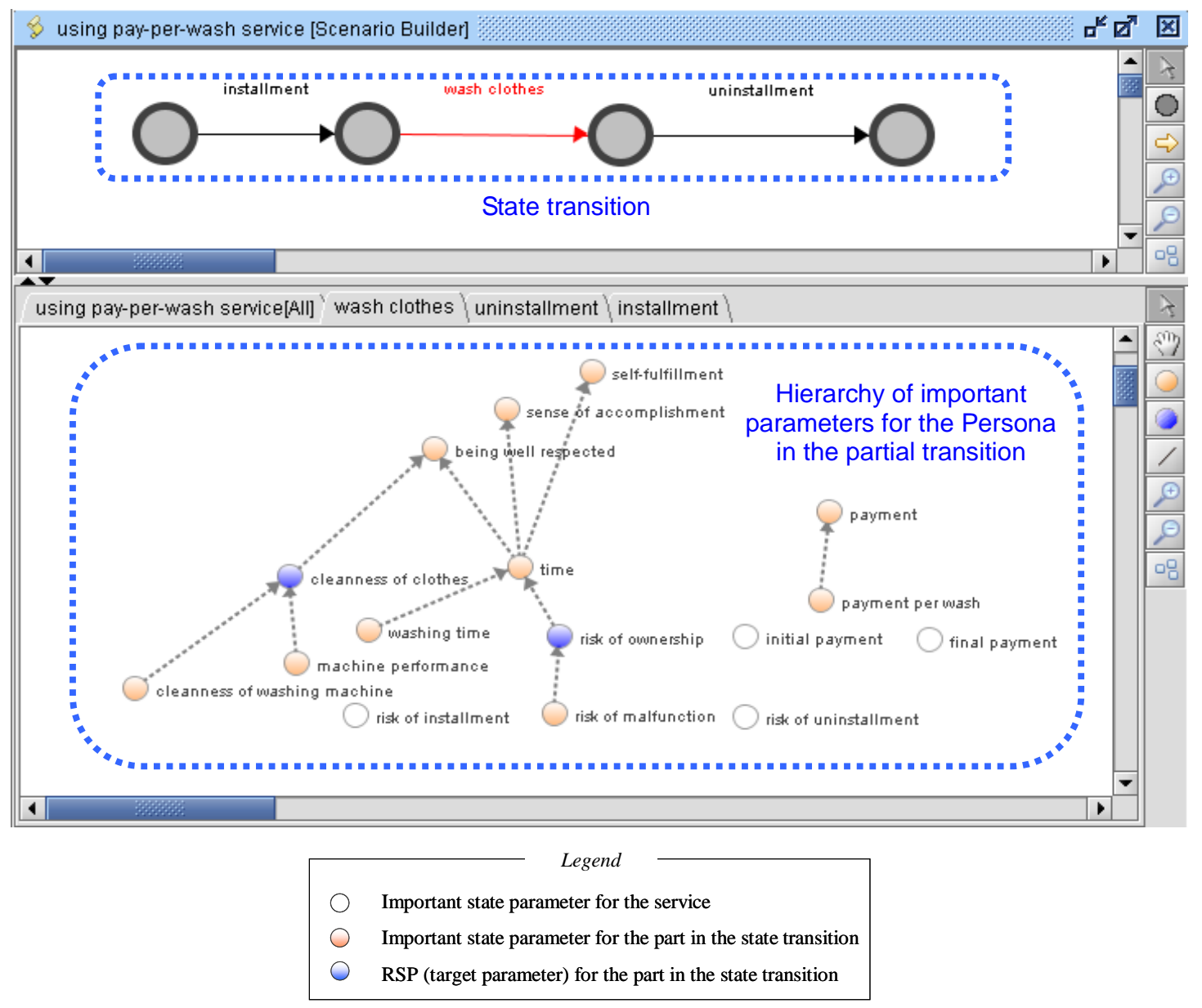

Fig. 16. A screen dump of extracting RSP for a Persona.

The flow model described in Service Explorer includes "service provider" (the washing-machine manufacturer in this case), the "electricity supplier", "water supplier", 
"detergent supplier", and "end user" (i.e. the end receiver) according to the service scheme described at the beginning of Section 4.2. The upper half of Fig. 17 shows this flow model. The already-identified state transition of the end receiver is shown in the lower half of Fig. 17 in a different form of that in Fig. 16. The end receiver's RSP, "cleanness of clothes" and "risk of ownership", in the action "wash clothes", are shown as well.

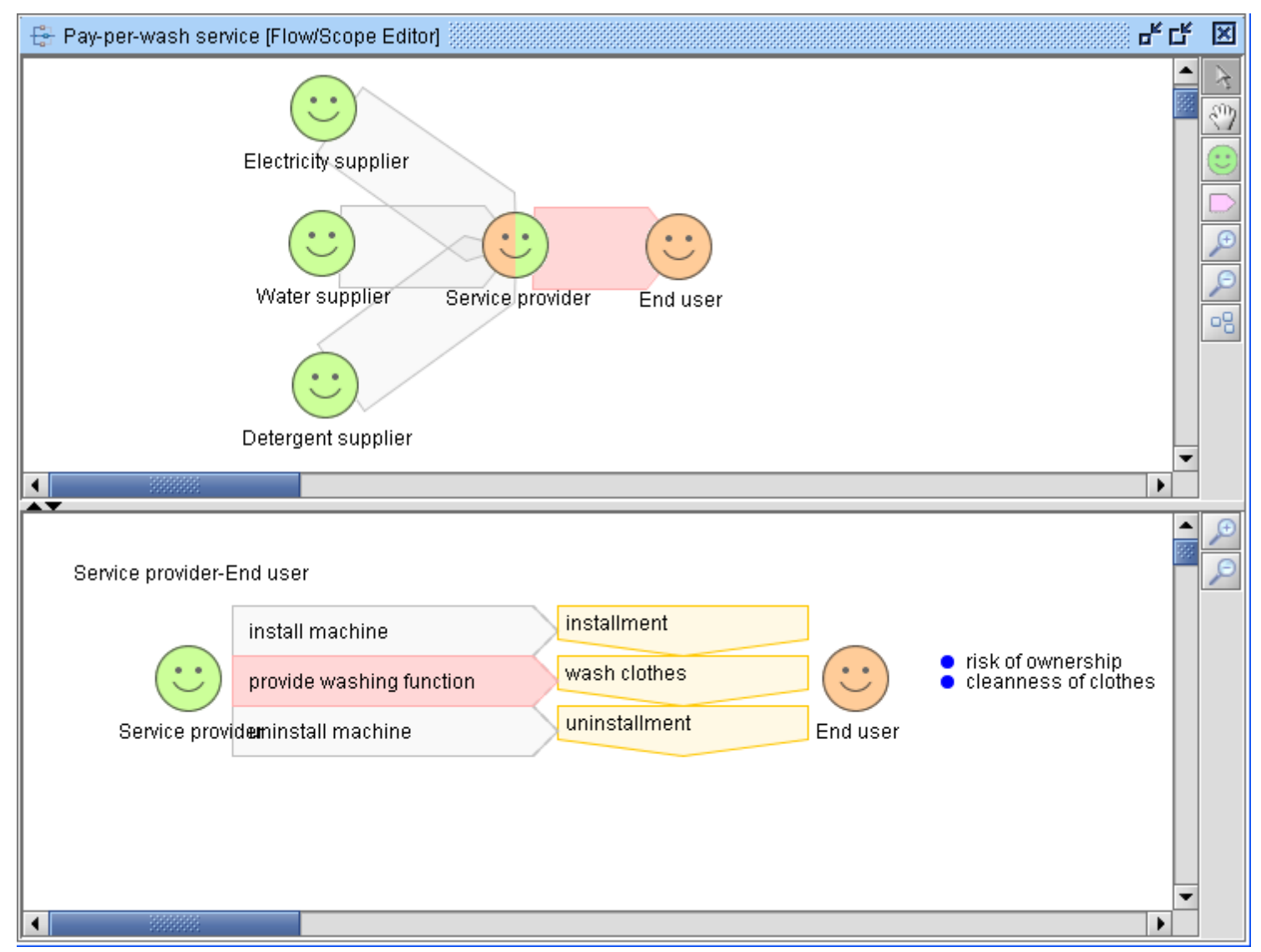

Fig. 17. Flow model and the scope model between the provider and the end user.

Fig. 18 shows the view model for the RSP "cleanness of clothes". This RSP was related to a CoP named "cleaning performance", whose associated function is "provide washing". The "provide washing" is deployed into "provide electricity", "provide water", "provide detergent", "provide maintenance" and "wash clothes". The first four functions are not needed in the case of a selling-typed service (described in Section 4.1). In addition, the entities related to those functions such as "electricity", "electricity meter", "detergent", "transporters" (meaning people transporting the detergent to the end users, thus the linked function "provide detergent" is the function of service activity), are newly introduced in this service as well. The parameters representing costs such as "cost of electricity" are included as ChP, because these are important for the provider who pays for them. It should be emphasized that the function, "wash clothes", and its lower parts (enclosed with blue dotted lines) are the same as the view model for "machine performance" in the case of a selling-typed service except for several links; e.g. the 
link between "Power consumption" of "Motor" and "cost of electricity". This suggests to designers that the power consumption of the motor should be, at least partially, considered negative to the service provider in this service. It should be recalled that the electricity cost is on the provider in this service. Another link is between "reliability" of "motor" and "malfunction frequency", which is further liked to "provide maintenance". Since maintenance by the engineers of the provider is included in the service with a flat price, this means the reliability becomes more important for the service provider in this service than in the selling-typed one.

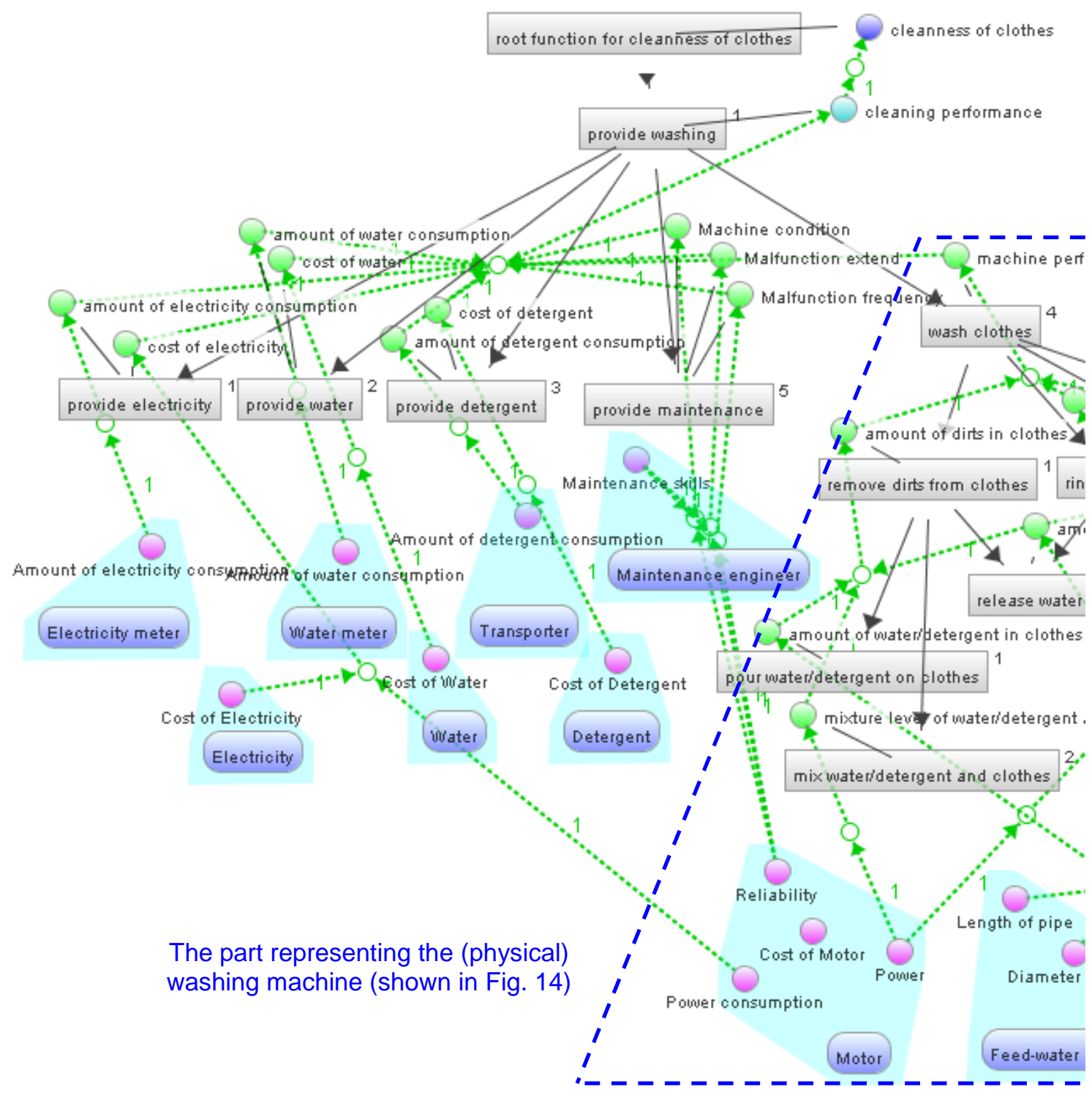

Fig. 18. The view model for "cleanness of clothes" (partial).

\subsection{Cleaning Washing Machines}

Let us assume that designers target a state parameter in the hierarchy shown at the far left hand-side in the lower half of Fig. 16, which is "cleanness of washing machine". This 
assumption is reasonable, because this parameter is a lower parameter, "cleanness of clothes" (the RSP in Fig. 18), but was not actually targeted in the pay-per-wash service. This can be applied not only to a pay-per-wash service, but also to a selling-type one. In fact, this is in operation in Japan for the selling-type service, which contributes also to operational efficiency of a maintenance base of a manufacturer. This service is effective because a type of fungus often grows inside a washing machine due to the high humid climate in Japan. It is critical that the inside is cleaned by disassembling the machine. Note that end receivers cannot disassemble it because they lack the knowledge about the product, and because the machine is not easy to disassemble. Then, a manufacturer's knowledge about its own products, as well as the type of products in general, is adopted as a source of the value created for this service.

Here, only a view model for the RSP, "cleanness of washing machine", is described as in Fig. 19. The top function is finally deployed into "clean outer parts", "disassemble washing machine", "clean tub", and "assemble washing-machine". The described FP include "amount of dirt on tub" and "knowledge of washing-machine". Since some FP are related to the washing machine, and some parts of a washing machine are described as entities, designers can understand that the washing machine functions as a channel. It should be emphasized that this surrounding service can be described partially using the same pieces of data used in other services shown in Sections 4.2 and 4.3. In this case, the entities such as the motor, casing and tub are shared.

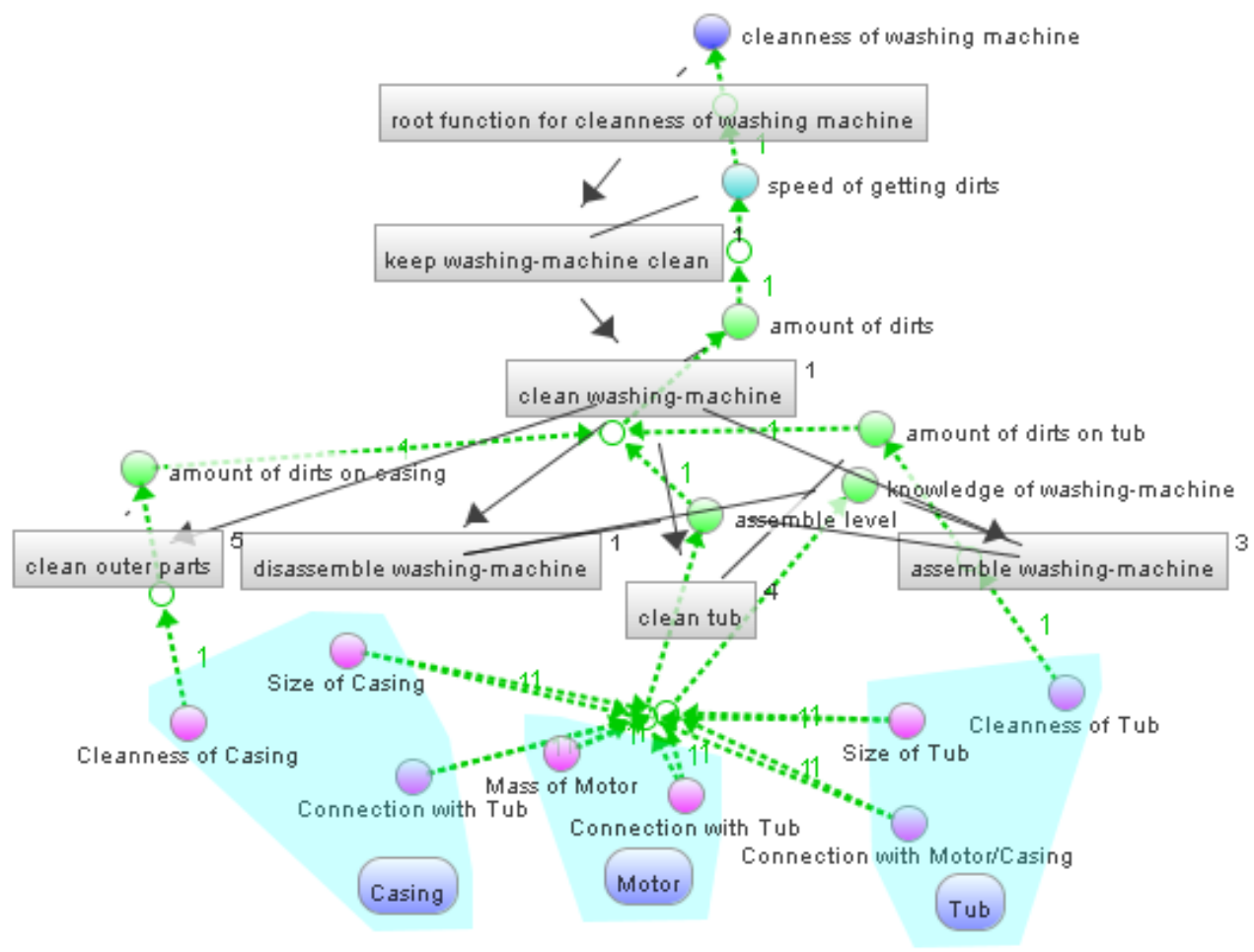

Fig. 19. The view model for the cleanness of the machine. 


\subsection{Verifying the Modeling}

The application of our model as explained in Sections 4.1, 4.2, and 4.3 proves that Service Explorer is able to describe the following.

1) Provided value

Service Explorer allows designers to describe the value of a receiver in a concerned service in the form of RSP. Thus, it explicitly represents a goal which is more abstract than the functionality which the designers traditionally aimed at. For instance, the view model in the pay-per-wash service (in Fig. 18) suggested that the value of the washing machine in that case is not the machine performance that is the value in the selling type, but rather the cleaning performance as explained in Section 4.2. This allows designers to search for a solution within a wider space.

In addition, the information grounding value can be modeled by the scenario model as well. Specifically, the hierarchy of important parameters reveal the base from which RSP are chosen. In addition, this provided a reason for a new service (cleaning washing machines) related to an existing one (cleaning clothes), as shown in Section 4.3. The Persona model was also proved to be effective to relate some of the important parameters at the highest level to the characteristics of the target receivers using the set of LOV. Other information in the form of free texts is effective for designers to share the concept of target receivers. It should be emphasized that the scenario model in the case in Section 4.2 worked for grounding the value, which was deployed into a set of physical products and service activities forming a new service.

2) Measures to provide value

Service Explorer allows designers to describe measures to realize the value of a receiver in the form of functions, which are generally used to understand the roles of products and service activities. For instance, the view model in Fig. 18 showed that the functions such as "wash clothes", "provide electricity" and "provide maintenance" are measures to provide the value of the service, as explained in Section 4.2. Furthermore, a physical product and a "service person" adopted in a service are described as an entity on such functions that are deployed enough as explained in 4.2. This allows designers to describe explicitly how a product or a service person as an entity functions so as to provide the intended value. In addition, the agents participating in the service are modeled using a flow model.

3) Relations among agents

Service Explorer enables us to describe the relations among agents participating in the service in the form of value/costs and their realization structures in a scope model. Thus, represented is the fundamental information: Who transports what to whom. In addition, the relations of agents in the service are qualitatively described. Such information is a trade-off relationship between agents and a contradiction within the whole service. For instance, when a parameter is increased, an agent becomes happier while another gets unhappier. Using this information, an initial provider, who designs the service, can attempt to find a solution on a trade-off relationship between agents and to solve a conflict within the whole service. This may be partially supported by the service evaluator, who identifies critical design parameters [31] so that we can select a relatively influential trade-off. For example, the capacity of the tub of a washing machine is in 
proportion to the value of the end user (machine performance) from the machine manufacturer in Fig. 13 for the selling-typed service; However, it is proportional to the cost of the retailer from the manufacturer as well. Such a relationship in a service has been out of the boundary of traditional product design.

As explained above, the information of what, why, who, and how shown in Table 1 were proved to be described by Service Explorer. In addition, the relations among agents can be modeled in a qualitative form. Nevertheless, it should be further investigated whether the described information of service is sufficient for designers. For instance, when information, e.g. procedures of service activities along the time axis with various inputs/outputs, may be described in more detail with the BPMN (Business Process Modeling Notation) Modeler [36, 37] according to a Service Blueprint [9], as proposed in another paper [38].

\section{Discussion}

The focus of this paper has been on service CAD systems required in SPE, which will be utilized early in the product/service development (i.e. in conceptual design). The prototype of the service CAD system named Service Explorer has been verified through the application of examples. It was revealed that Service Explorer covers the stages of conceptual design that have been neglected by most of the CAD systems. More specifically, using the models of Service Explorer, designers described and operated the design objects, as explained in Sections 4.1, 4.2 and 4.3.

The paper [10] also addressed Service Explorer and SPE so that it proposed a way to solve a problem that current Ecodesign (environmentally conscious design) techniques have. On the other hand, this paper focuses on implementation (more details on the modeling method and the information management) such as the CAD program. Thus, the paper [10] is an application paper in a specific theme, and addresses very little in the area of product design, while at the same time this paper is a fundamental contribution to basic insights of CAD theories and practices applicable not only to Ecodesign, but also to designing services/products in general. Issues regarding design for Product/Service Systems have been further considered in other studies (e.g. [39, 40]), while this paper has been focusing more on computer support in order to reach a well-designed product/service.

Service Explorer has high scalability due to its modular structure in the system, as well as its adoption of object-oriented programming. The modular structure, named "pluggable structure" [41], enables us to implement additional modules such as reasoning engines. In addition, the robustness of the system, meaning the robustness of the obtained solutions using the system, may be lower than that of conventional CAD systems for physical products due to the widened design space. However, using Service Explorer will contribute to attempting to keep the robustness by taking advantage of the power of describing/managing design objects.

The information described by Service Explorer, especially that regarding view models, could be utilized effectively so as to derive the relative importance of FP. This could be achieved with the QFD technique and the scores on RSP, and we have already begun the method development 
and implementation [31].

The current version of Service Explorer is based on functions as most traditional design tools are. However, the Persona model allows for more user (customer)-integrated design and more of the theories of mass customization (see e.g. [42]) and affordance-based design (e.g. [43]), which is based on perceptual psychology. As Brown and Blessing [44] conclude in their paper, affordance-based design is a useful complement to function-based design when dealing with "possible actions" by the users. The mindset of affordance-based design is an interesting follow-up, along with other design theories deriving from other fields (another instance of such research is seen in [45]) and will be considered in order to obtain as satisfying a service CAD system as possible. The modular design of Service Explorer makes this future development possible. One advantage of SPE as compared to affordance-based design is its friendliness to engineers, since they adapt the concept of functions that have been more familiar to them than concepts in the field of psychology.

There is an emerging research field called Service Science (short for Services Sciences, Management, and Engineering: SSME) [46, 47], which is the study of service systems including IT service systems. According to the increasing importance of services, they regard SSME as a seed becoming a new important discipline, although it is not yet well established as a full-fledged discipline as is for example computer science. The difference between SPE and SSME is that SPE addresses product design as an important element, while SSME does not.

As this service CAD system is useful in the early phases of conceptual design, it is important it be compatible with CAD systems further down the value chain (see e.g. [19]). This is also in line with the research conducted by Abrahamson et al. [20], and the standardization goals set by the National Institute of Standards and Technology (NIST) [21]. Service Explorer also has the possibility to be further connected with other CAD systems dealing with more concrete information such as functions and geometry, as it represents product functions and characteristics in view models as mentioned in Section 3.1.6.

How Service Explorer transforms the current process of SPE is summarized. Designers will share value of service receivers, which are explicitly represented, as well as the relative importance of such value in Service Explorer. Thus, they can avoid useless efforts to improve relatively unimportant value. In addition, they can share the structure of products and services to provide value in a systematic way, and can evaluate the structure quantitatively in a computational way using the service evaluator [31]. Furthermore, developing a new service can be more efficient by reusing the information of an existing service on Service Explorer. Service Explorer can help designers with generating new ideas by taking advantage of its computing power, e.g., use of Universal Abduction Studio (UAS) [35].

Future work includes implementation of the remaining parts of Service Explorer. More importantly, design procedures using the proposed model will be developed and implemented. These are in line with implications in other literature, such as in [48]. Specifically, a synthesis process with Service Explorer is to be developed. We will implement a methodology to obtain final solutions using a decision making process formalized in [49]. In addition, integration with other types of CAD systems, such as CAD for functional design and geometric design, will be 
needed to increase the effectiveness of SPE.

\title{
6. Conclusions
}

This paper proposed a new prototype of service CAD system called Service Explorer to support conceptual design in SPE. To do so, a design-object model was defined and the system was implemented as computer software. Service Explorer was verified through its application to several business cases. The model was proven to represent such properties that are crucial to achieve SPE effectively, such as value, costs, and functions, either of products or of service activities, and of entities.

\section{Acknowledgements}

We would like to express our gratitude to Mr. Tatsunori Hara and Prof. Tamio Arai from the Univ. of Tokyo for their cooperating implementation and discussion of Service Explorer. We also would like to thank the international PSS Design Research Community (www.pssdesignresearch.org) for the fruitful discussion. This research was also partially supported by a Research Fellowship Program by Alexander von Humboldt Foundation in Germany. The applications presented in Section 4 are based on the discussion with $A B$ Electrolux and Toshiba Service \& Engineering Co., Ltd.

\section{Notation list}

\author{
ChP: Channel Parameter \\ CoP: Content Parameter \\ DB: Database \\ FI: Function Influence \\ FN: Function Name \\ FP: Function Parameter \\ ICT: Information and Communication Technology \\ IT: Information Technology \\ MVC: Model View Controller \\ PSS: Product/Service-System \\ RSP: Receiver State Parameter \\ SPE: Service/Product Engineering \\ UAS: Universal Abduction Studio
}

\section{References}

[1] Oliva R, Kallenberg R. Managing the Transition from Products to Services. International Journal of Service Industry Management 2003; 14(2): 160-172.

[2] Chesbrough HW, Toward a New Science of Services, in Harvard Business Review. 2005. $20-21$.

[3] Japanese Ministry of Public Management, Home Affairs, Posts and Telecommunications, Posts and Telecommunications, Annual Report on the Family Income and Expenditure Survey. 2002: Japan Statistical Association.

[4] Alonso-Rasgado T, Thompson G, Elfstrom B. The Design of Functional (Total Care) Products. Journal of Engineering Design 2004; 15(6): 515-540.

[5] Tukker A, Tischner U. New Business for Old Europe. Sheffield: Greenleaf Publishing; 2006.

[6] Morelli N. Product-Service Systems, a Perspective Shift for Designers: A Case Study: The Design of 
a Telecentre. Design Studies 2003; 24(1): 73-99.

[7] Lindahl M, Ölundh G. The Meaning of Functional Sales. in 8th CIRP International Seminar on Life Cycle Engineering - Life Cycle Engineering: Challenges and Opportunities. 2001, 211-220.

[8] Schilling M. Strategic Management of Technological Innovation. Irwin: McGraw-Hill; 2004.

[9] Shostack GL, How to Design a Service, in Marketing of Services, J.H. Donnelly and W.R. George, Editors: American Marketing Association; 1981, 221-229.

[10] Sakao T, Shimomura Y. Service Engineering: A Novel Engineering Discipline for Producers to Increase Value Combining Service and Product. Journal of Cleaner Production 2007; 15(6): 590-604.

[11] Tomiyama T. Service Engineering to Intensify Service Contents in Product Life Cycles. in Second International Symposium on Environmentally Conscious Design and Inverse Manufacturing. Tokyo. IEEE Computer Society. 2001, 613-618.

[12] Ramaswamy R. Design and Management of Service Processes. Reading: MA: Addison-Wesley; 1996.

[13] Shimomura Y, Tomiyama T. Service Modeling for Service Engineering. in 5th International Conference on Design of Information Infrastructure Systems for Manufacturing. Osaka. 2002, 309-316.

[14] Bullinger H, Fahnrich K, Meiren T. Service Engineering - Methodical Development of New Service Products. International Journal of Production Economics 2003; 11: 275-287.

[15] Weber C, Steinbach M, Botta C, Deubel T. Modeling of Product-Service Systems (PSS) Based on the PDD Approach. in International Design Conference. Dubrovnik. 2004, 547-554.

[16] Stone RB, Chakrabarti A. Editors. Special Issue: Engineering Applications of Representations of Function, Part 1. Artificial Intelligence for Engineering Design, Analysis and Manufacturing 2005; 19(2): 63-132.

[17] Stone RB, Chakrabarti A. Editors. Special Issue: Engineering Applications of Representations of Function, Part 2. Artificial Intelligence for Engineering Design, Analysis and Manufacturing 2005; 19(3): 137-219.

[18]Fuh JYH, Li WD. Advances in Collaborative CAD: The-State-of-the Art. Computer-Aided Design 2005; 37(5): 571-581.

[19] Li WD, Lu WF, Fuh JYH, Wong YS. Collaborative Computer-Aided Design-Research and Development Status. Computer-Aided Design 2005; 37(9): 931-940.

[20] Abrahamson S, Wallace D, Senin N, Sferro P. Integrated Design in a Service Marketplace. Computer-Aided Design 2000; 32(2): 97-107.

[21] Sudarsan R, Fenves SJ, Sriram RD, Wang F. A Product Information Modeling Framework for Product Lifecycle Management. Computer-Aided Design 2005; 37(13): 1399-1411.

[22] Hoffman CM, Joan-Arinyo R. Cad and the Product Master Model. Computer-Aided Design 1998; 30(11): 905-918.

[23] Ullman DG. Toward the Ideal Mechanical Engineering Design Support System. Research in Engineering Design 2002; 13: 55-64.

[24] Arai T, Shimomura Y. Proposal of Service CAD System -a Tool for Service Engineering-. Annals of the CIRP 2004; 53(1): 397-400.

[25] Miles L. Techniques of Value Analysis and Engineering. USA: McGraw-Hill; 1971.

[26] Bolton RN, Drew JH. A Multistage Model of Customers' Assessments of Service Quality and Value. Journal of Consumer Research 1991; 17(March): 375-384.

[27] Cooper A. The Inmates Are Running the Asylum. USA: Sams; 1999.

[28] Pruitt J, Adlin T. The Persona Lifecycle: Keeping People in Mind Throughout Product Design. San Francisco: Morgan Kaufmann; 2006.

[29] Saaty L. The Analytic Hierarchy Process. USA: McGraw-Hill; 1980.

[30] Green E, Srinivasan V. Conjoint Analysis in Consumer Research: Issues and Outlook. Journal of Consumer Research 1978; 5: 103-123.

[31] Shimomura Y, Sakao T. A Service Evaluation Method for Service/Product Engineering. in International Conference on Engineering Design. Paris. 2007, CD ROM.

[32] Akao Y. Quality Function Deployment. Portland, OR: Productivity Press; 1990.

[33] Coyne R. Logic Models of Design. London: Pitman; 1998.

[34] Roozenburg NJM, Eekels J. Product Design: Fundamentals and Methods. Chichester, MA: John Wiley \& Sons; 1995.

[35] Takeda H, Sakai H, Nomaguchi Y, Yoshioka M, Shimomura Y, Tomiyama T. Universal Abduction Studio -Proposal of a Design Support Environment for Creative Thinking in Design-. in 14th 
International Conference on Engineering Design 2003. Stockholm. 2003, CD-ROM.

[36] Modeler EPSB. [cited; Available from: http://www.eclipse.org/stp/bpmn/] accessed on August 6, 2008.

[37] Information BPMNB. [cited; Available from: http://www.bpmn.org/] accessed on August 6, 2008.

[38] Hara T, Arai T, Shimomura Y, Sakao T, Service/Product Engineering: A New Discipline for Value Production, in 19th International Conference on Production Research. 2007, CD ROM.

[39] Sundin E, Lindahl M, Ijomah W. Product Design for Product/Service Systems - Design Experiences from Three Manufacturing Companies from Sweden. Journal of Engineering Design, in review.

[40] Sundin E, Bras B. Making Functional Sales Environmentally and Economically Beneficial through Product Remanufacturing. Journal of Cleaner Production 2005; 13(9): 913-925.

[41] Yoshioka M, Umeda Y, Takeda H, Shimomura Y, Nomaguchi Y, Tomiyama T. Physical Concept Ontology for the Knowledge Intensive Engineering Framework. Advanced Engineering Informatics 2004; 18(2): 95-113.

[42] Sundin E, Lindahl M, Comstock M, Shimomura Y, Sakao T, Integrated Product and Service Engineering Enabling Mass Customization, in 19th International Conference on Production Research. 2007, CD ROM.

[43] Maier JRA, Fadel GM. Comparing Function and Affordance as Bases for Design. in ASME 2002 Design Engineering Technical Conferences and Computers and Information in Engineering Conference. Montreal. 2002, CD-ROM.

[44] Brown DC, Blessing L. The Relationship between Function and Affordance. in ASME 2005 Design Engineering Technical Conferences and Computers and Information in Engineering Conference. Long Beach, California. 2005, CD-ROM.

[45] Vakili V, Chiu I, Shu LH, McAdams D, Stone R, Including Functional Models of Biological Phenomena as Design Stimuli, in ASME International Design Engineering Technical Conferences and Computers and Information in Engineering Conference. Las Vegas, USA. 2007, CD-ROM.

[46] National Innovation Initiative, Innovate America -Thriving in a World of Challenge and ChangeInterim Report. 2004: US Council on Competitiveness.

[47] Maglio PP, Keulen SSJT, Spohrer J, Service Systems, Service Scientists, SSME, and Innovation, in Communications of the ACM. 2006. 81-85.

[48] Sakao T, Sandström GÖ, Matzen D. Framing Design Research for Service Orientation through PSS Approaches. Journal of Engineering Design, in print.

[49] Sakao T, Watanabe K, Shimomura Y. A Method to Support Environmentally Conscious Service Design Using Quality Function Deployment (QFD). in Third International Symposium on Environmentally Conscious Design and Inverse Manufacturing. Tokyo. IEEE Computer Society. 2003, 567-574. 